

\title{
Categories of abelian varieties over finite fields, I: Abelian varieties over $\mathbb{F}_{p}$
}

\author{
Tommaso Giorgio Centeleghe and Jakob Stix
}

We assign functorially a $\mathbb{Z}$-lattice with semisimple Frobenius action to each abelian variety over $\mathbb{F}_{p}$. This establishes an equivalence of categories that describes abelian varieties over $\mathbb{F}_{p}$ avoiding $\sqrt{p}$ as an eigenvalue of the Frobenius in terms of simple commutative algebra. This result extends the isomorphism classification of Waterhouse and Deligne's equivalence for ordinary abelian varieties.

1. Introduction

2. On the ubiquity of Gorenstein rings among minimal central orders

3. Remarks on reflexive modules

4. Abelian varieties with minimal endomorphism algebra

5. Construction of the antiequivalence

6. Properties of the functor $T$

7. Ambiguity and comparison

\section{Introduction}

1.1. Let $p$ be a prime number, $\overline{\mathbb{F}}_{p}$ an algebraic closure of the prime field $\mathbb{F}_{p}$ with $p$ elements, and $\mathbb{F}_{q} \subset \overline{\mathbb{F}}_{p}$ the subfield with $q$ elements, where $q=p^{e}$ is a power of $p$. The category

$$
\mathrm{AV}_{q}
$$

of abelian varieties over $\mathbb{F}_{q}$ is an additive category, where for any two objects $A, B$ the abelian group $\operatorname{Hom}_{\mathbb{F}_{q}}(A, B)$ is free of finite rank. Even though the main result of this paper concerns abelian varieties over the prime field $\mathbb{F}_{p}$, the general theme of our work is describing suitable subcategories $\mathrm{C}$ of $\mathrm{AV}_{q}$ by means of lattices $T(A)$

Centeleghe was supported by a project jointly funded by the DFG Priority Program SPP 1489 and the Luxembourg FNR.

MSC2010: 11-02.

Keywords: abelian varieties, finite fields, Gorenstein rings, reflexive modules. 
functorially attached to abelian varieties $A$ of $\mathrm{C}$. In contrast to the characteristic-zero case, if we insist that

$$
\operatorname{rk}_{\mathbb{Z}}(T(A))=2 \operatorname{dim}(A),
$$

then it is not possible to construct $T(A)$ on the whole category $\mathrm{AV}_{q}$ (see Section 1.6). However, if we take $C$ to be the full subcategory

$$
\mathrm{AV}_{q}^{\text {ord }}
$$

of ordinary abelian varieties, Deligne $[1969, \S 7]$ showed that a functor $A \mapsto T(A)$ satisfying (1-1) exists and gives an equivalence between $\mathrm{AV}_{q}^{\text {ord }}$ and the category of finite free $\mathbb{Z}$-modules $T$ equipped with a linear map $F: T \rightarrow T$ satisfying a list of easy-to-state axioms.

Inspired by Waterhouse [1969, Theorem 6.1], in the present work we show that a description in the style of Deligne can in fact be obtained, when $q=p$, for a considerably larger subcategory $\mathrm{C}$ of $\mathrm{AV}_{p}$, which excludes only a single isogeny class of simple objects of $\mathrm{AV}_{p}$ from occurring as an isogeny factor (see Theorem 1). Deligne's method is an elegant application of the Serre-Tate theory of canonical liftings of ordinary abelian varieties, whereas our method, closer to that used by Waterhouse, does not involve lifting abelian varieties to characteristic zero. Even if the main result of this paper generalizes the $q=p$ case of Deligne's theorem, it is unlikely that a proof generalizing Deligne's lifting strategy is possible.

1.2. A Weil $q$-number $\pi$ is an algebraic integer, lying in some unspecified field of characteristic zero, such that for any embedding $\iota: \mathbb{Q}(\pi) \hookrightarrow \mathbb{C}$ we have

$$
|\iota(\pi)|=q^{1 / 2},
$$

where $|-|$ is the ordinary absolute value of $\mathbb{C}$. Two Weil $q$-numbers $\pi$ and $\pi^{\prime}$ are conjugate to each other if there exists an isomorphism $\mathbb{Q}(\pi) \stackrel{\sim}{\longrightarrow} \mathbb{Q}\left(\pi^{\prime}\right)$ carrying $\pi$ to $\pi^{\prime}$, in which case we write $\pi \sim \pi^{\prime}$. We will denote by $W_{q}$ the set of conjugacy classes of Weil $q$-numbers. A Weil $q$-number is either totally real or totally imaginary, hence it makes sense to speak of a nonreal element of $W_{q}$.

Let $A$ be an object of $\mathrm{AV}_{q}$, and denote by $\pi_{A}: A \rightarrow A$ the Frobenius isogeny of $A$ relative to $\mathbb{F}_{q}$. If $A$ is $\mathbb{F}_{q}$-simple then $\operatorname{End}_{\mathbb{F}_{q}}(A) \otimes \mathbb{Q}$ is a division ring, and a well-known result of Weil says that $\pi_{A}$ is a Weil $q$-number inside the number field $\mathbb{Q}\left(\pi_{A}\right)$. Let

$$
A \sim \prod_{1 \leq i \leq r} A_{i}^{e_{i}}
$$

be the decomposition of $A$ up to $\mathbb{F}_{q}$-isogeny into powers of simple, pairwise nonisogenous factors $A_{i}$. The Weil support of $A$ is defined as the subset

$$
w(A)=\left\{\pi_{A_{1}}, \ldots, \pi_{A_{r}}\right\} \subseteq W_{q}
$$


given by the conjugacy classes of the Weil numbers $\pi_{A_{i}}$ attached to the simple factors $A_{i}$. By Honda-Tate theory, the conjugacy classes of the $\pi_{A_{i}}$ are pairwise distinct; moreover, any class in $W_{q}$ arises as $\pi_{A}$, for some $\mathbb{F}_{q}$-simple abelian variety $A$, uniquely determined up to $\mathbb{F}_{q}$-isogeny [Tate 1971, Théorème 1].

1.3. Consider now the case $q=p$. Using Honda-Tate theory, it is easy to see that for a simple object $A$ of $\operatorname{AV}_{p}$ the ring $\operatorname{End}_{\mathbb{F}_{p}}(A)$ is commutative if and only if $\pi_{A} \nsucc \sqrt{p}$, i.e., if and only if the Frobenius isogeny $\pi_{A}: A \rightarrow A$ defines a nonreal Weil $p$-number [Waterhouse 1969, Theorem 6.1]. Let

$$
\mathrm{AV}_{p}^{\mathrm{com}}
$$

be the full subcategory of $\mathrm{AV}_{p}$ given by all objects $A$ such that $w(A)$ does not contain the conjugacy class of $\sqrt{p}$. Equivalently, $\mathrm{AV}_{p}^{\text {com }}$ is the largest full subcategory of $\mathrm{AV}_{p}$ closed under taking cokernels containing all simple objects whose endomorphism ring is commutative. Since the Weil $p$-number $\sqrt{p}$ is associated to an $\mathbb{F}_{p}$-isogeny class of simple, supersingular abelian surfaces [Tate 1971, Exemple (b), p. 97], we have a natural inclusion $\mathrm{AV}_{p}^{\text {ord }} \subset \mathrm{AV}_{p}^{\text {com }}$.

The main result of this paper, proven at the end of Section 5.3, is the following: Theorem 1. There is an ind-representable contravariant functor

$$
A \mapsto(T(A), F)
$$

which induces an antiequivalence between $\mathrm{AV}_{p}^{\text {com }}$ and the category of pairs $(T, F)$ given by a finite, free $\mathbb{Z}$-module $T$ and an endomorphism $F: T \rightarrow T$ satisfying the following properties:

(i) $F \otimes \mathbb{Q}$ is semisimple, and its eigenvalues are nonreal Weil p-numbers.

(ii) There exists a linear map $V: T \rightarrow T$ such that $F V=p$.

Moreover, the lattice $T(A)$ has rank $2 \operatorname{dim}(A)$ for all $A$ in $\mathrm{AV}_{p}^{\text {com }}$, and $F$ is equal to $T\left(\pi_{A}\right)$.

To prove the theorem, we consider in Section 2 a family of Gorenstein rings

$$
R_{w}=\mathbb{Z}[F, V] /\left(F V-p, h_{w}(F, V)\right)
$$

indexed by the finite subsets $w \subseteq W_{p}$, where $h_{w}(F, V)$ is a certain symmetric polynomial built out of the minimal polynomials over $\mathbb{Q}$ of the elements of $w$. An object $(T, F)$ in the target category of the functor $T(-)$ of Theorem 1 is nothing but an $R_{w}$-module, for $w \subset W_{p}$ large enough, that is free of finite rank as a $\mathbb{Z}$ module. In this translation, the linear map $F: T \rightarrow T$ is given by the action of the image of $F$ in $R_{w}$, and the relation $h_{w}(F, V)$ in $R_{w}$ encodes precisely that $F \otimes \mathbb{Q}$ acts semisimply and with eigenvalues given by Weil $p$-numbers lying in $w$ (see Sections $2.4,2.5$ and 3.2). Thanks to the Gorenstein property, these $R_{w}$-modules 
are precisely the reflexive $R_{w}$-modules; the category that they form will be denoted by (see Section 3)

$$
\operatorname{Refl}\left(R_{w}\right) .
$$

For $v \subseteq w$, the corresponding rings are linked by natural surjective maps $\operatorname{pr}_{v, w}: R_{w} \rightarrow R_{v}$. We denote by $\Re_{p}^{\text {com }}$ the pro-system $\left(R_{w}, \mathrm{pr}_{v, w}\right)$ with $w \subseteq W_{p}$ ranging over the finite subsets avoiding the conjugacy class of $\sqrt{p}$. We further set

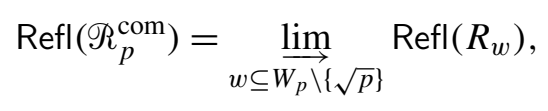

and refer to Section 3.2 for details.

In this language, Theorem 1 can be stated as saying that

$$
T: \mathrm{AV}_{p}^{\mathrm{com}} \rightarrow \operatorname{Refl}\left(\Re_{p}^{\mathrm{com}}\right)
$$

is an antiequivalence of categories. While this formulation of the main result is closer to the perspective we adopted in its proof, the more concrete statement we chose to give above allows an immediate comparison to Deligne's result [1969].

1.4. The rings $R_{w}$ studied in Section 2 are in fact defined for any finite subset $w \subseteq W_{q}$. They appear naturally in connection to abelian varieties, in that for any $A$ in $\mathrm{AV}_{q}$ the natural map

$$
\mathbb{Z}[F, V] /(F V-q) \rightarrow \operatorname{End}_{\mathbb{F}_{q}}(A)
$$

sending $F$ to $\pi_{A}$ and $V$ to the Verschiebung isogeny $q / \pi_{A}$ induces an identification between $R_{w(A)}$ and the subring $\mathbb{Z}\left[\pi_{A}, q / \pi_{A}\right]$ of $\operatorname{End}_{\mathbb{F}_{q}}(A)$, which has finite index in the center (see Section 2.1). The rings $R_{w}$ have been already considered in [Waterhouse 1969] and [Howe 1995], for example. The Gorenstein property of $R_{w}$ in the ordinary cases is implicitly contained in [Howe 1995] and explicitly used in a special case in [Howe 2004]. However, to the best of our knowledge, a systematic investigation of the occurrence of Gorensteinness among the rings $R_{w}$ has not been carried out previously (see Theorems 11 and 12).

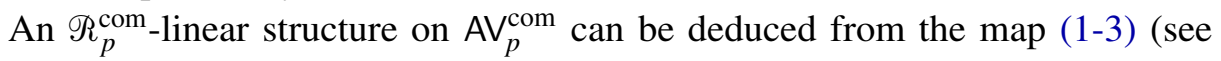
Section 2.3). The requirement that $F=T\left(\pi_{A}\right)$ means precisely that the functor

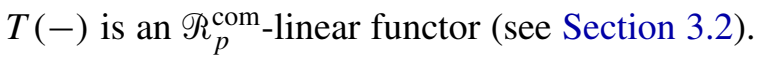

1.5. The proof of the theorem consists of two steps. First, for any finite subset $w \subseteq W_{p}$ not containing the conjugacy class of $\sqrt{p}$, we construct a certain abelian variety $A_{w}$ isogenous to the product of all simple objects attached to the elements of $w$ via Honda-Tate theory. The object $A_{w}$ is chosen in its isogeny class with the smallest possible endomorphism ring, i.e., such that the natural map

$$
R_{w} \rightarrow \operatorname{End}_{\mathbb{F}_{p}}\left(A_{w}\right)
$$


is an isomorphism (see Proposition 21). In order to show the existence of such an $A_{w}$, which already appears in [Waterhouse 1969, Theorem 6.1] if $w$ consists of a single element, the assumption $q=p$ plays an important role. Exploiting the Gorenstein property of $R_{w}$, in Theorem 25 we show that the functor $\operatorname{Hom}_{\mathbb{F}_{p}}\left(-, A_{w}\right)$ gives a contravariant equivalence

$$
\operatorname{Hom}_{\mathbb{F}_{p}}\left(-, A_{w}\right): \mathrm{AV}_{w} \stackrel{\sim}{\longrightarrow} \operatorname{Refl}\left(R_{w}\right),
$$

where $\mathrm{AV}_{w}$ is the full subcategory of $\mathrm{AV}_{p}$ given by all abelian varieties $A$ with $w(A) \subseteq w$.

The second step consists in showing that the abelian varieties $A_{w}$ previously constructed can be chosen in such a way that the functors $\operatorname{Hom}_{\mathbb{F}_{p}}\left(-, A_{w}\right)$ interpolate well and define a functor on $\mathrm{AV}_{p}^{\mathrm{com}}$. More precisely we show the existence of an ind-system

$$
\mathscr{A}=\left(A_{w}, \varphi_{w, v}\right),
$$

indexed by finite subsets $w \subseteq W_{p}$ not containing the conjugacy class of $\sqrt{p}$, such that the corresponding direct limit of finite free $\mathbb{Z}$-modules

$$
T(A)=\underset{w}{\lim } \operatorname{Hom}_{\mathbb{F}_{p}}\left(A, A_{w}\right)
$$

stabilizes for any $A$ in $\mathrm{AV}_{p}^{\mathrm{com}}$. The contravariant functor $T(-)$ ind-represented by $A$ will produce the required antiequivalence.

1.6. As Serre has observed, it is not possible to functorially construct a lattice $T(A)$ satisfying the expected $\mathrm{rk}_{\mathbb{Z}}(T(A))=2 \operatorname{dim}(A)$ on the category of abelian varieties over $\overline{\mathbb{F}}_{p}$. This is due to the existence of objects like supersingular elliptic curves $E$ over $\overline{\mathbb{F}}_{p}$. As is well known, the division ring $\operatorname{End}_{\overline{\mathbb{F}}_{p}}(E) \otimes \mathbb{Q}$ is a nonsplit quaternion algebra over $\mathbb{Q}$ and has no 2-dimensional $\mathbb{Q}$-linear representation that can serve as $T(E) \otimes \mathbb{Q}$. The issue just described is the same obstruction that prevents the existence of a Weil cohomology for varieties over finite fields with rational coefficients.

Using the same argument, one can show the nonexistence of a lattice $T(A)$ as above on the category $\mathrm{AV}_{q}$, where $q$ is a square. When $q$ is not a square, the correct instance of Serre's observation preventing Theorem 1 from extending to all of $\mathrm{AV}_{p}$ is given by the isogeny class of $\mathbb{F}_{q}$-simple, supersingular abelian surfaces associated via Honda-Tate theory to the real, nonrational, Weil $q$-number $\sqrt{q}$. The endomorphism ring of any such surface $A$ is an order of a quaternion algebra over $\mathbb{Q}(\sqrt{q})=\mathbb{Q}(\sqrt{p})$ which is ramified at the two real places [Waterhouse 1969, p. 528]. It follows that $\operatorname{End}_{\mathbb{F}_{q}}(A) \otimes \mathbb{R} \simeq \mathbb{U} \times \mathbb{W}$ is a product of two copies of the Hamilton quaternions $\mathbb{W}$. Thus it admits no faithful representation on a 4-dimensional real vector space, which $T(A) \otimes \mathbb{R}$ would give rise to. 
1.7. The dual abelian variety establishes an antiequivalence $A \mapsto A^{t}$ of $\mathrm{AV}_{q}$ which preserves Weil supports and has the effect of switching the roles of Frobenius and Verschiebung endomorphisms relative to $\mathbb{F}_{q}$. That is,

$$
\left(\pi_{A}\right)^{t}=q / \pi_{A^{t}}
$$

as isogenies from $A^{t}$ to itself. On the module side, we define a covariant involution of $\operatorname{Refl}\left(\Re_{p}^{\text {com }}\right)$, denoted by $M \mapsto M^{\tau}$, which interchanges the roles of $F$ and $V$, i.e., such that

$$
(T, F)^{\tau}=(T, p / F) .
$$

Using these two dualities we can exhibit a covariant version of the functor $T(-)$ of Theorem 1. More precisely, define

$$
T_{*}(A)=T\left(A^{t}\right)^{\tau}
$$

as the pair given by the $\mathbb{Z}$-module $T\left(A^{t}\right)$ equipped with the linear map $p / T\left(\pi_{A^{t}}\right)$. In the notation as pairs, $T_{*}(A)$ takes the form

$$
\left(T\left(A^{t}\right), p / T\left(\pi_{A^{t}}\right)\right)=\left(T\left(A^{t}\right), T\left(\left(\pi_{A}\right)^{t}\right)\right)=\left(T_{*}(A), T_{*}\left(\pi_{A}\right)\right) .
$$

The functor $T_{*}(-)$ gives a covariant, $\mathscr{R}_{p}^{\mathrm{com}}$-linear equivalence

$$
T_{*}: \mathrm{AV}_{p}^{\mathrm{com}} \rightarrow \operatorname{Refl}\left(\mathscr{R}_{p}^{\mathrm{com}}\right)
$$

which is pro-represented by the system $\mathscr{A}^{t}=\left(A_{w}^{t}, \varphi_{w^{\prime}, w}^{t}\right)$ dual to (1-4). In the definition of $T_{*}(-)$ it is necessary to apply the involution $\tau$ to $T\left(A^{t}\right)$ in order to guarantee that $T_{*}$ be $\mathscr{R}_{p}^{\text {com-linear. }}$

In Section 7.4 we compare $T_{*}(-)$ restricted to $\mathrm{AV}_{p}^{\text {ord }}$ with Deligne's functor [1969, $\S 7]$, which we denote by $T_{\mathrm{Del}, p}(-)$. The comparison makes use of a compatible pro-system of projective $R_{w}$-modules $M_{w}$ of rank 1 for all finite subsets $w \subseteq W_{p}$ consisting only of conjugacy classes of ordinary Weil $p$-numbers. Proposition 44 then describes, for all abelian varieties $A$ over $\mathbb{F}_{p}$ with $w(A) \subseteq w$, a natural isomorphism

$$
T_{\text {Del }, p}(A) \otimes_{R_{w}} M_{w} \stackrel{\sim}{\rightarrow} T_{*}(A) .
$$

Furthermore, by choosing a suitable ind-representing system $\mathscr{A}=\left(A_{w}, \varphi_{v, w}\right)$, we may assume that $M_{w}=R_{w}$ for all $w$, i.e., the antiequivalence of Theorem 1 may be chosen in its covariant version to extend Deligne's equivalence; see Proposition 45 for details.

1.8. Finally, we indicate how to recover the $\ell$-adic Tate module $T_{\ell}(A)$, for a prime $\ell \neq p$, and the contravariant Dieudonné module $T_{p}(A)$ (see [Waterhouse 1969, §1.2]) from the module $T(A)$. This involves working with the formal Tate module $T_{\ell}(\mathscr{A})$ and the formal Dieudonné module $T_{p}(\mathscr{A})$ of the direct system $\mathscr{A}$, respectively 
defined as the direct limit of $T_{\ell}\left(A_{w}\right)$ and the inverse limit of the $T_{p}\left(A_{w}\right)$, with transition maps obtained via functoriality of $T_{\ell}$ and $T_{p}$. More concretely, we have natural isomorphisms

$$
\begin{aligned}
& T_{\ell}(A) \simeq \operatorname{Hom}_{\mathscr{R}_{\ell}}\left(T(A) \otimes \mathbb{Z}_{\ell}, T_{\ell}(\mathscr{A})\right), \\
& T_{p}(A) \simeq\left(T(A) \otimes \mathbb{Z}_{p}\right) \widehat{\otimes}_{\mathscr{R}_{p}} T_{p}(\mathscr{A}) ;
\end{aligned}
$$

see Propositions 27 and 28 for notation and proofs. In this respect the functor $T(-)$ can be interpreted as an integral lifting of the Dieudonné module functor $T_{p}(-)$.

In a forthcoming paper, we will apply the method used here to study certain categories of abelian varieties over a finite field which is larger that $\mathbb{F}_{p}$. Therefore, although Theorem 1 deals with abelian varieties over $\mathbb{F}_{p}$, we only restrict to the case $q=p$ when it becomes necessary.

\section{On the ubiquity of Gorenstein rings among minimal central orders}

2.1. Minimal central orders. Let $w \subseteq W_{q}$ be any finite set of conjugacy classes of Weil $q$-numbers. Choose Weil $q$-numbers $\pi_{1}, \ldots, \pi_{r}$ representing the elements of $w$, and consider the ring homomorphism

$$
\mathbb{Z}[F, V] /(F V-q) \rightarrow \prod_{1 \leq i \leq r} \mathbb{Q}\left(\pi_{i}\right)
$$

sending $F$ to $\left(\pi_{1}, \ldots, \pi_{r}\right)$ and $V$ to $\left(q / \pi_{1}, \ldots, q / \pi_{r}\right)$.

Definition 2. The minimal central order $R_{w}$ is the quotient

$$
\mathbb{Z}[F, V] /(F V-q) \rightarrow R_{w}
$$

by the kernel of the homomorphism (2-1). The image of $F$ in $R_{w}$ will be denoted by $F_{w}$, and the image of $V$ by $V_{w}$.

The construction of the ring $R_{w}$ is independent of the chosen Weil $q$-numbers in their respective conjugacy classes. When $w$ consists of a single conjugacy class of a Weil number $\pi$, the ring $R_{\{\pi\}}$, isomorphic to the order of $\mathbb{Q}(\pi)$ generated by $\pi$ and $q / \pi$, will sometimes be denoted simply by $R_{\pi}$. Since the representatives $\pi_{1}, \ldots, \pi_{r}$ are pairwise nonconjugate, there is a canonical finite index inclusion

$$
R_{w} \subseteq \prod_{\pi \in w} R_{\pi}
$$

in particular,

$$
R_{w} \otimes \mathbb{Q}=\prod_{\pi \in w} \mathbb{Q}(\pi) .
$$

Moreover, for finite subsets $v \subseteq w \subseteq W_{q}$ we have a natural surjection

$$
\operatorname{pr}_{v, w}: R_{w} \rightarrow R_{v} \text {. }
$$


Our main goal in this section is to show that, under a mild assumption on $w$, the ring $R_{w}$ is a 1-dimensional Gorenstein ring. This will be proved in Section 2.5, where we obtain a description of $R_{w}$ by identifying the relations between the generators $F$ and $V$.

Example 3. The equality of closed subschemes

$$
\operatorname{Spec}\left(R_{w}\right)=\bigcup_{\pi \in w} \operatorname{Spec}\left(R_{\pi}\right) \subseteq \operatorname{Spec}(\mathbb{Z}[F, V] /(F V-q))
$$

shows that the spectrum of $R_{w}$ is obtained by gluing the spectra of the rings $R_{\pi}$ along their various intersections inside $\operatorname{Spec}(\mathbb{Z}[F, V] /(F V-q))$. This means, roughly, that congruences between Weil $q$-numbers are responsible for $R_{w}$ differing from the product of the $R_{\pi}$ for all $\pi \in w$.

We measure in a special situation the deviation of $R_{w}$ from being isomorphic to $\prod_{\pi \in w} R_{\pi}$. For $i=1,2$, let $\pi_{i}$ be a quadratic Weil $q$-number with minimal polynomial

$$
x^{2}-\beta_{i} x+q,
$$

where $\beta_{i} \in \mathbb{Z}$, and set $\Delta=\beta_{1}-\beta_{2}$. Since $q / \pi_{i}=\beta_{i}-\pi_{i}$, we have

$$
R_{\pi_{i}}=\mathbb{Z}\left[\pi_{i}\right] \simeq \mathbb{Z}[x] /\left(x^{2}-\beta_{i} x+q\right) ;
$$

moreover, the subring $R_{w} \subseteq \mathbb{Z}\left[\pi_{1}\right] \times \mathbb{Z}\left[\pi_{2}\right]$ is generated as a $\mathbb{Z}$-algebra by

$$
(0, \Delta),\left(\pi_{1}, \pi_{2}\right) \in \mathbb{Z}\left[\pi_{1}\right] \times \mathbb{Z}\left[\pi_{2}\right],
$$

since it is generated by $\left(\pi_{1}, \pi_{2}\right)$ and $\left(\beta_{1}-\pi_{1}, \beta_{2}-\pi_{2}\right)$. Because $\beta_{1} \equiv \beta_{2}$ modulo $\Delta$, there are isomorphisms of quotients

$$
\mathbb{Z}\left[\pi_{1}\right] / \Delta \mathbb{Z}\left[\pi_{1}\right] \simeq \mathbb{Z}\left[\pi_{2}\right] / \Delta \mathbb{Z}\left[\pi_{2}\right]=: R_{0},
$$

and $R_{w}$ becomes the fiber product

$$
R_{w}=\mathbb{Z}\left[\pi_{1}\right] \times_{R_{0}} \mathbb{Z}\left[\pi_{2}\right],
$$

which is an order of index $\Delta^{2}$ in the product $R_{\pi_{1}} \times R_{\pi_{2}}$. The congruences between $\pi_{1}$ and $\pi_{2}$ are encoded by the closed subscheme of $\operatorname{Spec}(\mathbb{Z}[F, V] /(F V-q))$ given by

$$
\operatorname{Spec}\left(R_{0}\right)=\operatorname{Spec}\left(R_{\pi_{1}}\right) \cap \operatorname{Spec}\left(R_{\pi_{2}}\right) .
$$

Note that the minimal polynomials $x^{2}-\beta_{i}+q$ yield Weil $q$-numbers if and only if

$$
\beta_{i}^{2}<4 q \text {. }
$$

In particular, by letting $q$ range over the powers of the prime $p$, the Weil $q$-numbers $\pi_{i}$ may be chosen so that $\Delta$ is divisible by an arbitrary integer. 
2.2. Connection to abelian varieties. We proceed to link $R_{w}$ to abelian varieties over $\mathbb{F}_{q}$. Any such $A$ has two distinguished isogenies, given by the Frobenius $\pi_{A}$ and the Verschiebung $q / \pi_{A}$ relative to $\mathbb{F}_{q}$. The $\mathbb{Q}$-algebra $\operatorname{End}_{\mathbb{F}_{q}}(A) \otimes \mathbb{Q}$ is semisimple, and its center is equal to the subalgebra $\mathbb{Q}\left(\pi_{A}\right)$ generated by $\pi_{A}$ [Tate 1966, Theorem 2]. It follows that any isogeny decomposition of $A$, as in (1-2), induces the isomorphism

$$
\mathbb{Q}\left(\pi_{A}\right) \simeq \prod_{\pi_{A_{i}} \in w(A)} \mathbb{Q}\left(\pi_{A_{i}}\right)
$$

sending $\pi_{A}$ to $\left(\pi_{A_{1}}, \ldots, \pi_{A_{r}}\right)$, where $\pi_{A_{1}}, \ldots, \pi_{A_{r}}$ are the Weil $q$-numbers defined by the simple factors of $A$ and $w(A)$ is the Weil support of $A$ defined in the introduction.

From (2-4) we deduce that the ring homomorphism

$$
r_{A}: \mathbb{Z}[F, V] /(F V-q) \rightarrow \operatorname{End}_{\mathbb{F}_{q}}(A)
$$

sending $F$ to $\pi_{A}$ and $V$ to $q / \pi_{A}$ gives an identification between $R_{w(A)}$ and the image of $r_{A}$, namely the subring

$$
\mathbb{Z}\left[\pi_{A}, q / \pi_{A}\right]
$$

which sits inside the center of $\operatorname{End}_{\mathbb{F}_{q}}(A)$ with finite index. In this way we see that $R_{w(A)}$ plays the role of a lower bound for the center of $\operatorname{End}_{\mathbb{E}_{q}}(A)$. This justifies the terminology we chose in its definition.

Remark 4. One can ask whether there exists an abelian variety $A$ with Weil support $w$ such that the natural map $R_{w} \rightarrow \operatorname{End}_{\mathbb{F}_{p}}(A)$ induced by $r_{A}$ gives an isomorphism between $R_{w}$ and the center of $\operatorname{End}_{\mathbb{F}_{p}}(A)$. In Proposition 21 below, generalizing a result of Waterhouse, we obtain a partial result in this direction.

2.3. Linear structures over minimal central orders. For a finite subset $w \subseteq W_{q}$ the full subcategory

$$
\mathrm{AV}_{w} \subseteq \mathrm{AV}_{q}
$$

consists of all abelian varieties $A$ such that $w(A) \subseteq w$ or, equivalently, such that $r_{A}$ factors through the quotient $\mathbb{Z}[F, V] /(F V-q) \rightarrow R_{w}$. Since for any morphism $f: A \rightarrow B$ in $\mathrm{AV}_{q}$ and any $\eta \in \mathbb{Z}[F, V] /(F V-q)$ the diagram

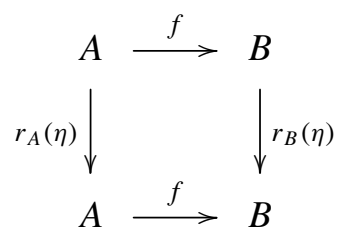


is commutative, as follows from the naturality of the Frobenius and Verschiebung isogenies, we deduce an $R_{w}$-linear structure on the category $\mathrm{AV}_{w}$. Furthermore, for finite subsets $v \subseteq w$ the $R_{w}$-linear structure on $\mathrm{AV}_{v}$ induced by the fully faithful inclusion $\mathrm{AV}_{v} \subseteq \mathrm{AV}_{w}$ is compatible, via the surjection $\mathrm{pr}_{v, w}$, with the $R_{v}$-linear structure on $\mathrm{AV}_{v}$.

Remark 5. If $W \subseteq W_{q}$ is now any subset, denote by $\mathscr{R}_{W}$ the projective system $\left(R_{w}, \mathrm{pr}_{w, v}\right)$ as $w$ ranges through all finite subsets of $W$, and by $\mathrm{AV}_{W}$ the full subcategory of $\mathrm{AV}_{q}$ whose objects are all abelian varieties $A$ with $w(A) \subseteq W$. We will treat $\mathrm{AV}_{W}$ as the direct 2-limit of the categories $\mathrm{AV}_{w}$, for finite subsets $w$ of $W$. The collection of $R_{w}$-linear structures on the subcategories $\mathrm{AV}_{w} \subseteq \mathrm{AV}_{W}$, which are linked by the compatibility conditions described above, form what we will refer to as the $\mathscr{R}_{W}$-linear structure on $\mathrm{AV}_{W}$.

2.4. The symmetric polynomial. Let $\pi$ be a Weil $q$-number. If $\mathbb{Q}(\pi)$ has a real place then $\pi^{2}=q$, so that $\mathbb{Q}(\pi)$ is totally real, and $[\mathbb{Q}(\pi): \mathbb{Q}]$ is either 2 or 1 according to whether the degree $e=\left[\mathbb{F}_{q}: \mathbb{F}_{p}\right]$ is odd or even, respectively. In the first case there is only one conjugacy class of real Weil $q$-numbers; in the second one there are two of them, given by the rational integers $q^{e / 2}$ and $-q^{e / 2}$. In the general case where $\pi$ is not real, the field $\mathbb{Q}(\pi)$ is a nonreal CM field, with complex conjugation induced by $\pi \mapsto q / \pi$.

The degree $2 d=[\mathbb{Q}(\pi): \mathbb{Q}]$ is even, except for the two rational Weil $q$-numbers occurring for $e$ even, in which case $d=1 / 2$. Set

$$
P_{\pi}(x)=x^{2 d}+a_{2 d-1} x^{2 d-1}+\cdots+a_{1} x+a_{0} \in \mathbb{Z}[x]
$$

for the normalized minimal polynomial of $\pi$ over $\mathbb{Q}$. The polynomial $P_{\pi}(x)$ depends only on the conjugacy class of $\pi$. The following lemma is well known (see [Howe 1995, Proposition 3.4]):

Lemma 6. Let $\pi$ be a nonreal Weil $q$-number. For $r \geq 0$, we have $a_{d-r}=q^{r} a_{d+r}$.

Proof. We can arrange the roots $\alpha_{1}, \ldots, \alpha_{2 d}$ of $P_{\pi}(x)$ so that $\alpha_{i}$ and $\alpha_{2 d+1-i}$ are complex conjugates of each other, that is, $\alpha_{i} \alpha_{2 d+1-i}=q$. For a subset $I \subseteq\{1, \ldots, 2 d\}$ we set $I^{c}=\{1, \ldots, 2 d\} \backslash I$ and $\bar{I}=\{i: 2 d+1-i \in I\}$; we will use the multiindex notation $\alpha^{I}=\prod_{i \in I} \alpha_{i}$. Then, summing over subsets of $\{1, \ldots, 2 d\}$, we compute

$$
\begin{aligned}
(-1)^{d+r} a_{d-r} & =\sum_{|I|=d+r} \alpha^{I}=\left(\prod_{i=1}^{2 d} \alpha_{i}\right) \cdot \sum_{|I|=d+r} \frac{1}{\alpha^{I^{c}}} \\
& =q^{r} \cdot \sum_{|J|=d-r} \frac{q^{d-r}}{\alpha^{J}}=q^{r} \cdot \sum_{|J|=d-r} \alpha^{\bar{J}}=q^{r}(-1)^{d-r} a_{d+r} .
\end{aligned}
$$


We next construct a symmetric polynomial $h_{\pi}(F, V) \in \mathbb{Z}[F, V]$. The idea is to consider the rational function $P_{\pi}(F) / F^{d} \in \mathbb{Z}[F, q / F]$ (at least when $d \in \mathbb{Z}$ ), and then formally set $V=q / F$.

Definition 7. We define the symmetric polynomial $h_{\pi}(F, V)$ attached to a Weil $q$-number $\pi$ as follows:

(1) If $\pi$ is a nonreal Weil $q$-number, then we set $h_{\pi}(F, V)=F^{d}+a_{2 d-1} F^{d-1}+\cdots+a_{d+1} F+a_{d}+a_{d+1} V+\cdots+a_{2 d-1} V^{d-1}+V^{d}$.

(2) If $\pi= \pm p^{m} \sqrt{p}$ is real but not rational, then we set

$$
h_{\pi}(F, V)=F-V .
$$

(3) If $\pi= \pm p^{m}$ is rational, then we set

$$
h_{ \pm p^{m}}(F, V)=F^{1 / 2} \mp V^{1 / 2} .
$$

The polynomial $h_{w}(F, V)$ just defined belongs to $\mathbb{Z}[F, V]$ if $\pi$ not rational, and to $\mathbb{Z}\left[F^{1 / 2}, V^{1 / 2}\right]$ otherwise. It appears already in [Howe 1995, §9].

Lemma 8. (1) If $\pi$ is a nonreal Weil $q$-number, then we have $h_{\pi}(\pi, q / \pi)=0$.

(2) If $\pi$ is a real, but not rational, Weil $q$-number, then $h_{\pi}(F, V)=F-V$ and $h_{\pi}(\pi, q / \pi)=0$.

(3) If $\pi= \pm p^{m}$ is rational, then $h_{p^{m}}(F, V) \cdot h_{-p^{m}}(F, V)=F-V$ is again contained in $\mathbb{Z}[F, V]$, and vanishes for $F=\pi$ and $V=q / \pi$.

Proof. Assertion (1) follows from $h_{\pi}(\pi, q / \pi)=P_{\pi}(\pi) / \pi^{d}=0$ which is based on Lemma 6. Assertion (2) and (3) are trivial.

Definition 9. An ordinary Weil $q$-number is a Weil $q$-number $\pi$ such that exactly half of the roots of its minimal polynomial $P_{\pi}(x)$ in an algebraic closure of $\mathbb{Q}_{p}$ are $p$-adic units.

A Weil $q$-number is ordinary if and only if its associated isogeny class of simple abelian varieties over $\mathbb{F}_{q}$ is ordinary. Real Weil numbers are not ordinary.

Lemma 10. Let $w \subseteq W_{q}$ be a finite subset of nonreal conjugacy classes of Weil $q$-numbers. Then $w$ consists of ordinary conjugacy classes if and only if $h_{w}(0,0)$ is not divisible by $p$.

Proof. Let $\alpha_{1}, \ldots, \alpha_{d}, q / \alpha_{1}, \ldots, q / \alpha_{d}$ be the roots of $\prod_{\pi \in w} P_{\pi}(x)$. Then

$$
h_{w}(F, V) \equiv \prod_{i=1}^{d}\left(F-\left(\alpha_{i}+q / \alpha_{i}\right)+V\right) \quad \bmod (F V-q)
$$


so that

$$
h_{w}(F, V) \equiv(-1)^{d} \prod_{i=1}^{d}\left(\alpha_{i}+q / \alpha_{i}\right) \quad \bmod p .
$$

This integer is not divisible by $p$ if and only if the algebraic integers $\alpha_{i}+q / \alpha_{i}$ are $p$-adic units for all $i$. This happens if and only if either $\alpha_{i}$ or $q / \alpha_{i}$ are $p$-adic units for all $i$, that is, if $w$ consists of ordinary conjugacy classes.

2.5. Structure of the minimal central orders. In what follows we will define the degree of a finite subset $w \subseteq W_{q}$ by

$$
\operatorname{deg}(w)=\operatorname{rk}_{\mathbb{Z}}\left(R_{w}\right)=\sum_{\pi \in w}[\mathbb{Q}(\pi): \mathbb{Q}] .
$$

So $w$ is of even degree if and only if $w$ either contains none or both rational Weil $q$-numbers $\pm q^{e / 2}$, which only exist when $e=\left[\mathbb{F}_{q}: \mathbb{F}_{p}\right]$ is even. Extending this notion, we will say that an arbitrary subset $W \subseteq W_{q}$ is of even degree if either none or both rational conjugacy classes of Weil $q$-numbers belong to $W$.

If $w \subseteq W_{q}$ is any finite subset, we set

$$
h_{w}(F, V)=\prod_{\pi \in w} h_{\pi}(F, V),
$$

which is contained in $\mathbb{Z}[F, V]$ as soon as $w$ is of even degree.

Theorem 11. Let $w \subseteq W_{q}$ be a finite set of Weil q-numbers of even degree.

(1) We have $R_{w}=\mathbb{Z}[F, V] /\left(F V-q, h_{w}(F, V)\right)$.

(2) The ring $R_{w}$ is a 1-dimensional complete intersection; in particular, it is a Gorenstein ring.

When $w$ consists of ordinary Weil $q$-numbers, part (1) of Theorem 11 is [Howe 1995, Proposition 9.1].

Proof. The ring $R_{w}$ is reduced as it injects into a product of number fields. Moreover, $R_{w}$ is a finite $\mathbb{Z}$-algebra, because it is generated by $F$ and $V$ satisfying integral relations in $R_{w}$. Thus $R_{w}$ is free of finite rank as a $\mathbb{Z}$-module and of Krull dimension 1. More precisely, by (2-3) we have

$$
\operatorname{rk}_{\mathbb{Z}}\left(R_{w}\right)=\sum_{\pi \in w}[\mathbb{Q}(\pi): \mathbb{Q}]=: 2 D
$$

The ring $\mathbb{Z}[F, V] /(F V-q)$ is a normal ring with at most one rational singularity in $\mathfrak{p}=(F, V, p)$. Hence, $h_{w}(F, V)$ is a nonzero divisor in $\mathbb{Z}[F, V] /(F V-q)$ and it remains to show (1) to conclude the proof of (2). 
We now show assertion (1). By Lemma 8 the evaluation of $h_{w}(F, V)$ in $R_{\pi}$ vanishes for all $\pi \in w$. Hence we obtain a surjection

$$
\varphi: S=\mathbb{Z}[F, V] /\left(F V-q, h_{w}(F, V)\right) \rightarrow R_{w} .
$$

We are done if we can show that $S$ is generated by $2 D$ elements as a $\mathbb{Z}$-module.

By construction, $h_{w}(F, V)$ is a product of polynomials of the form

$$
f_{\pi}(F)+g_{\pi}(V)
$$

with $f_{\pi}, g_{\pi} \in \mathbb{Z}[X]$ monic (or $-g_{\pi}$ monic). The degrees are $\operatorname{deg}\left(f_{\pi}\right)=\operatorname{deg}\left(g_{\pi}\right)=$ $[\mathbb{Q}(\pi): \mathbb{Q}] / 2$ if $\pi$ is nonrational, and 1 if $\pi$ is rational. Having a representative of the form $f(F)+g(V)$ for monic polynomials $f, g$ (or $-g$ ) of the same degree is preserved under taking products:

$\left(f_{1}(F)+g_{1}(V)\right)\left(f_{2}(F)+g_{2}(V)\right)$

$=f_{1} f_{2}(F)+g_{1} g_{2}(V)+$ lower degree terms in $F, V$,

where the mixed terms are of lower degree, because $F V=q$ necessarily leads to cancellations.

Hence the same holds for the product: $h_{w}(F, V)=f(V)+g(V)$ with $\operatorname{deg}(f)=$ $\operatorname{deg}(g)=D$. In particular,

$$
F^{D}, F^{D-1}, \ldots, F, 1, V, \ldots, V^{D-1}
$$

generate $S$ as a $\mathbb{Z}$-module.

Since Theorem 1 deals with abelian varieties over $\mathbb{F}_{p}$, our main concern in this paper are the commutative algebra properties of $R_{w}$ for finite subsets of $W_{p}$. Here Theorem 11 covers all cases. In order to complete the picture, we answer what happens if $w \subseteq W_{q}$ contains exactly one rational conjugacy class of Weil $q$-numbers.

Theorem 12. Let $q$ be the square of a positive or negative integer $\sqrt{q} \in \mathbb{Z}$. Let $v \subseteq W_{q}$ be a finite set containing no rational conjugacy class, and set $w=v \cup\{\sqrt{q}\}$.

(1) We have $R_{w}=\mathbb{Z}[F, V] /\left(F V-q, h_{v}(F, V)(F-\sqrt{q}), h_{v}(F, V)(V-\sqrt{q})\right)$.

(2) The ring $R_{w}$ is Gorenstein if and only if all conjugacy classes of Weil q-numbers in $v$ are ordinary.

Proof. Reasoning as in the proof of Lemma 8, we see that the defining quotient map $\mathbb{Z}[F, V] /(F V-q) \rightarrow R_{w}$ factors as a surjective map

$$
S=\mathbb{Z}[F, V] /\left(F V-q, h_{v}(F, V)(F-\sqrt{q}), h_{v}(F, V)(V-\sqrt{q})\right) \rightarrow R_{w} .
$$

As in Theorem 11, as a $\mathbb{Z}$-module, the ring $R_{w}$ is free of rank

$$
\operatorname{rk}_{\mathbb{Z}}\left(R_{w}\right)=1+\sum_{\pi \in v}[\mathbb{Q}(\pi): \mathbb{Q}]=: 2 D+1 .
$$


It is easy to see that $S$ is generated as a $\mathbb{Z}$-module by

$$
F^{D}, F^{D-1}, \ldots, F, 1, V, \ldots, V^{D} .
$$

This shows assertion (1) above.

For assertion (2), we first note that after inverting one of the elements $p, F$ or $V$, the three relations can be reduced to two relations, so that outside of ( $p, F, V)$ the ring $R_{w}$ is a local complete intersection and hence Gorenstein. It remains to discuss the local ring in $\mathfrak{p}=(p, F, V)$.

There is a unique polynomial $h \in \mathbb{Z}[X]$ such that

$$
h_{v}(F, V)=h(F)-h(0)+h(V) \in \mathbb{Z}[F, V],
$$

and for this $h$ we have $h(0)=h_{v}(0,0)$. Since $\mathbb{Z}$ is regular (hence Gorenstein) and $R_{w}$ is a flat $\mathbb{Z}$-algebra, it follows from [Matsumura 1989, Theorem 23.4] that $R_{w}$ is Gorenstein in $\mathfrak{p}$ if and only if

$$
R_{w} / p R_{w}=\mathbb{F}_{p}[F, V] /(F V, h(F) F, h(V) V)
$$

is Gorenstein in $\overline{\mathfrak{p}}=(F, V)$. The ring $R_{w} / p R_{w}$ is Artinian, hence of dimension 0 , so that by [Matsumura 1989, Theorem 18.1] the ring $\left(R_{w} / p R_{w}\right)_{\bar{p}}$ is Gorenstein if and only if

$$
1=\operatorname{dim}_{\mathbb{F}_{p}} \operatorname{Hom}\left(\kappa(\overline{\mathfrak{p}}), R_{w} / p R_{w}\right) .
$$

The space of homomorphisms has the same dimension as the socle, i.e., the maximal submodule annihilated by $(F, V)$. The socle is the intersection of the kernels of $F$ and $V$ as $\mathbb{F}_{p}$-linear maps of $R_{w}$, which can be easily evaluated in the basis $F^{D}, F^{D-1}, \ldots, F, 1, V, \ldots, V^{D}$. The intersection is 1 -dimensional if $p \nmid h(0)$, and it is 2-dimensional otherwise. By Lemma 10, this completes the proof.

\section{Remarks on reflexive modules}

3.1. Reflexive versus $\mathbb{Z}$-free. Let $S$ be a noetherian ring. Recall that a finitely generated $S$-module $M$ is reflexive (resp.torsionless) if the natural map

$$
M \rightarrow \operatorname{Hom}_{S}\left(\operatorname{Hom}_{S}(M, S), S\right)
$$

is an isomorphism (resp. injective). We denote the category of finitely generated reflexive $S$-modules by $\operatorname{Refl}(S)$.

Lemma 13. Let $w \subseteq W_{q}$ be a finite set of Weil q-numbers such that $R_{w}$ is Gorenstein, and let $\ell$ be a prime number. Let $M$ be a finitely generated $R_{w}$-module (resp. $R_{w} \otimes \mathbb{Z}_{\ell}$-module). The following are equivalent:

(a) $M$ is reflexive. 
(b) $M$ is torsionless.

(c) $M$ is free as a $\mathbb{Z}$-module (resp. $\mathbb{Z}_{\ell}$-module).

Proof. Assertions (a) and (b) are equivalent by [Bass 1963, Theorem 6.2(4)], since $R_{w}$ is Gorenstein and of dimension 1.

For a uniform treatment, we set $S=R_{w} \otimes \Lambda$ with $\Lambda=\mathbb{Z}$ (resp. $\Lambda=\mathbb{Z}_{\ell}$ ). Since $S$ is finite flat over $\Lambda$, the dual module $\operatorname{Hom}_{S}(M, S)$ is free as a $\Lambda$-module. The same holds for every submodule of $\operatorname{Hom}_{S}(M, S)$, which shows assertion (b) implies (c).

For the converse direction we introduce the total ring of fractions $S \subset K=S \otimes_{\mathbb{Z}} \mathbb{Q}$, which is a product of fields. Therefore, assuming (c), the composite map

$$
M \rightarrow M \otimes_{\mathbb{Z}} \mathbb{Q}=M \otimes_{S} K \rightarrow \operatorname{Hom}_{S}\left(\operatorname{Hom}_{K}\left(M \otimes_{S} K, K\right), K\right)
$$

is injective. And since it factors over the natural map $M \rightarrow \operatorname{Hom}_{S}\left(\operatorname{Hom}_{S}(M, S), S\right)$, the latter is also injective and hence $M$ is torsionless.

3.2. The main theorem with reflexive modules. Let $w \subseteq W_{q}$ be a finite set of conjugacy classes of Weil $q$-numbers of even degree (see Section 2.5), so that, in particular, $R_{w}$ is Gorenstein (see Theorem 11). For an object $M$ of $\operatorname{Refl}\left(R_{w}\right)$, let $\left(M_{0}, F_{M}\right)$ be the pair consisting of the $\mathbb{Z}$-module $M_{0}$ underlying $M$ and of the linear map $F_{M}: M_{0} \rightarrow M_{0}$ given by the action of $F_{w} \in R_{w}$ on $M$.

Proposition 14. The functor $M \mapsto\left(M_{0}, F_{M}\right)$ gives an equivalence between $\operatorname{Refl}\left(R_{w}\right)$ and the category of pairs $(T, F)$ consisting of a finite, free $\mathbb{Z}$-module $T$, and an endomorphism $F: T \rightarrow T$ satisfying the following conditions:

(i) $F \otimes \mathbb{Q}$ is semisimple with eigenvalues given by Weil $q$-numbers in $w$.

(ii) There exists $V: T \rightarrow T$ such that $F V=q$.

A morphism between two such pairs $(T, F)$ and $\left(T^{\prime}, F^{\prime}\right)$ is a linear map $f: T \rightarrow T^{\prime}$ such that $f F=F^{\prime} f$.

Proof. Thanks to Lemma 13, an $R_{w}$-module belongs to $\operatorname{Refl}\left(R_{w}\right)$ if and only if it is finite and free as a $\mathbb{Z}$-module. Moreover, the linear map $F_{M}: M_{0} \rightarrow M_{0}$ satisfies in the ring $\operatorname{End}_{\mathbb{Z}}\left(M_{0}\right)$ the polynomial

$$
F^{d} \cdot h_{w}(F, q / F)=\prod_{\pi \in w} P_{\pi}(F),
$$

which is squarefree. Therefore $F_{M} \otimes \mathbb{Q}$ is semisimple with eigenvalues given by Weil $q$-numbers whose conjugacy classes belong to $w$. The map $V_{M}: M_{0} \rightarrow M_{0}$ induced by the action of $V_{w} \in R_{w}$ on $M$ satisfies $V_{M} F_{M}=q$. Essential surjectivity of the functor follows easily from Lemma 13 . 
Let now $v \subseteq w$ be a finite subset which is also of even degree. By Lemma 13, the natural projection $\operatorname{pr}_{v, w}: R_{w} \rightarrow R_{v}$ gives a fully faithful embedding

$$
\operatorname{Refl}\left(R_{v}\right) \subseteq \operatorname{Refl}\left(R_{w}\right),
$$

by means of which $\operatorname{Refl}\left(R_{v}\right)$ can be regarded as the full subcategory whose objects are those for which the $R_{w}$-action factors over $\mathrm{pr}_{v, w}: R_{w} \rightarrow R_{v}$. Using the description of Proposition 14, we easily see that an object $M$ of $\operatorname{Refl}\left(R_{w}\right)$ lies in $\operatorname{Refl}\left(R_{v}\right)$ if and only if the eigenvalues of $F_{M} \otimes \mathbb{Q}: M_{0} \otimes \mathbb{Q} \rightarrow M_{0} \otimes \mathbb{Q}$ define conjugacy classes of Weil $q$-numbers in $v$.

Definition 15. Let $W \subseteq W_{q}$ be a subset of even degree, and $\mathscr{R}_{W}=\left(R_{w}\right)$ be the pro-ring with $w$ ranging over all finite subsets of $W$ of even degree. The category

$$
\operatorname{Refl}\left(\mathscr{R}_{W}\right):=\underset{w \subseteq W}{\lim _{w}} \operatorname{Refl}\left(R_{w}\right)
$$

is the full subcategory of the category of $\mathbb{Z}[F, V]$-modules given by all $M$ such that:

(1) There exists $w_{M} \subseteq W$ such that the structural action of $\mathbb{Z}[F, V]$ on $M$ factors through $\mathbb{Z}[F, V] \rightarrow R_{w_{M}}$ (and hence through $\mathbb{Z}[F, V] \rightarrow R_{w}$ for all $w$ containing $w_{M}$ ).

(2) For any finite $w \subseteq W$ of even degree containing $w_{M}$, the module $M$ is reflexive as an $R_{w}$-module.

Notice that condition (2) is equivalent to asking that $M$ be a reflexive module over $R_{w}$ for some $w \subseteq W$ of even degree such that the action of $R_{w}$ on $M$ is defined (see Lemma 13).

Remark 16. For any finite $w \subseteq W$ of even degree, the category Refl( $\left.\mathscr{R}_{W}\right)$ contains the $R_{w}$-linear category $\operatorname{Refl}\left(R_{w}\right)$ as a full subcategory. Moreover, if $v \subseteq w$ are finite subsets of $W$ of even degree, then the $R_{v}$-linear structure on $\operatorname{Refl}\left(R_{v}\right)$ induced from the fully faithful embedding $\operatorname{Refl}\left(R_{v}\right) \subseteq \operatorname{Refl}\left(R_{w}\right)$ is compatible, via the surjection $\mathrm{pr}_{v, w}: R_{w} \rightarrow R_{v}$, with the natural $R_{w}$-linear structure. Formally we are in a situation analogous to that described in Remark 5, where the category $\mathrm{AV}_{W}$ played the role of $\operatorname{Refl}\left(\mathscr{R}_{W}\right)$. We will then refer to this data as the $\mathscr{R}_{W}$-linear structure of $\operatorname{Refl}\left(\mathscr{R}_{W}\right)$.

The category $\operatorname{Refl}\left(\mathscr{R}_{W}\right)$ can be given a concrete description in terms of pairs $(T, F)$ given by a finite free $\mathbb{Z}$-module $T$ and a linear map $F: T \rightarrow T$ such that:

(i) $F \otimes \mathbb{Q}$ is semisimple and its eigenvalues are Weil $q$-numbers in $W$.

(ii) There exists $V: T \rightarrow T$ with $F V=q$.

The notion of morphism between two such pairs is clear. This can be seen reasoning as in Proposition 14, and using the compatibility of linear structures described in Remark 16. 
Denote now the set $W_{p} \backslash\{\sqrt{p}\}$ of nonreal conjugacy classes of Weil $p$-numbers simply by $W_{p}^{\text {com }}$, and the corresponding pro-ring $\mathscr{R}_{W_{p}^{\text {com }}}$ by $\mathscr{R}_{p}^{\text {com }}$. Theorem 1 then claims the existence of a contravariant, $\mathscr{R}_{W_{p}^{\text {com }}}$-linear, ind-representable equivalence

$$
T: \mathrm{AV}_{p}^{\mathrm{com}} \rightarrow \operatorname{Refl}\left(\mathscr{R}_{p}^{\mathrm{com}}\right)
$$

such that $T(A)$ is a lattice of rank $2 \operatorname{dim}(A)$. By definition, the $\mathscr{R}_{W_{p}^{\text {com }}}$-linearity of $T(-)$ is the requirement that for any finite $w \subseteq W_{p}^{\text {com }}$ the restriction of $T$ to $\mathrm{AV}_{w}$ has values in $\operatorname{Refl}\left(R_{w}\right)$ and is $R_{w}$-linear. These conditions amount precisely to the equality $F=T\left(\pi_{A}\right)$ for all $A$ in $\mathrm{AV}_{p}^{\text {com. }}$.

3.3. Further remarks. The following piece of homological algebra is used later:

Lemma 17. Let $S$ be a 1-dimensional Gorenstein ring. For any finitely generated reflexive $S$-module $M$, we have

$$
\operatorname{Ext}_{S}^{1}(M, S)=0 .
$$

Proof. We use a free presentation of the dual $\operatorname{Hom}_{S}(M, S)$ and dualize again. This yields an embedding of $M$ into a free $S$-module and then a short exact sequence

$$
0 \longrightarrow M \longrightarrow S^{n} \longrightarrow M^{\prime} \longrightarrow 0 .
$$

The Ext-sequence, and the fact that $S$ has injective dimension 1 [Bass 1963, §1], yield

$$
0=\operatorname{Ext}_{S}^{1}\left(S^{n}, S\right) \longrightarrow \operatorname{Ext}_{S}^{1}(M, S) \longrightarrow \operatorname{Ext}_{S}^{2}\left(M^{\prime}, S\right)=0
$$

from which the lemma follows.

Finally, here is a criterion for invertible reflexive modules in terms of their endomorphism algebras:

Proposition 18. Let $S$ be a reduced Gorenstein ring of dimension at most 1, and let $M$ be a reflexive module. Then the following are equivalent:

(a) $M$ is locally free of rank 1.

(b) The natural map $S \rightarrow \operatorname{End}_{S}(M)$ is an isomorphism.

Proof. If $M$ is locally free of $\operatorname{rank} 1$, then $\operatorname{End}_{S}(M) \simeq M^{\vee} \otimes M \simeq S$, where $M^{\vee}=\operatorname{Hom}_{S}(M, S)$, and (b) holds.

For the converse, we may assume that $S$ is a complete local ring by passing to the completion. Since $\operatorname{End}_{S}(M)=S$ we have $M \neq 0$, and, moreover, $M$ cannot be a module (extending the $S$-module structure) for a strictly larger subring of the total ring of fractions of $S$. Now [Bass 1963, Proposition 7.2] shows that $M$ has a nonzero projective direct summand $M_{0}$. With $M=M_{0} \oplus M_{1}$, we find

$$
S \times \operatorname{End}_{S}\left(M_{1}\right)=\operatorname{End}_{S}\left(M_{0}\right) \times \operatorname{End}_{S}\left(M_{1}\right) \subseteq \operatorname{End}_{S}(M)=S
$$


and therefore $\operatorname{End}_{S}\left(M_{1}\right)=0$. This forces $M_{1}=0$, and $M$ is projective. Then $\operatorname{End}_{S}(M)$ is projective with rank equal to the square of the rank of $M$ (as a locally constant function on $\operatorname{Spec}(S)$ ). Thus $M$ is of rank 1 and the proof is complete.

\section{Abelian varieties with minimal endomorphism algebra}

Before restricting to the case $q=p$, we recall the following classical result of Tate (see [Tate 1966, $\S 1$ ] for $\ell \neq p$, [Waterhouse and Milne 1971, Theorem 6] for any $\ell$, also [Chai et al. 2014, §A.1]) which will be used frequently. For $A$ an abelian variety over $\mathbb{F}_{q}$ and $\ell$ a prime number, denote by $A\left[\ell^{\infty}\right]$ the $\ell$-divisible group corresponding to $A$.

Theorem 19 (Tate). Let $A, B$ be abelian varieties over $\mathbb{F}_{q}$, and $\ell$ a prime number. The natural map $f \mapsto f\left[\ell^{\infty}\right]$ induces an isomorphism

$$
\operatorname{Hom}_{\mathbb{F}_{q}}(A, B) \otimes \mathbb{Z}_{\ell} \stackrel{\sim}{\rightarrow} \operatorname{Hom}\left(A\left[\ell^{\infty}\right], B\left[\ell^{\infty}\right]\right) .
$$

As is well known, the isomorphism of Tate's theorem takes a more concrete form as follows. If $\ell \neq p$, it can be formulated in terms of Galois representations, and says that the functor $\ell$-adic Tate module $T_{\ell}(-)$ induces an isomorphism

$$
\operatorname{Hom}_{\mathbb{F}_{q}}(A, B) \otimes \mathbb{Z}_{\ell} \stackrel{\sim}{\longrightarrow} \operatorname{Hom}_{\mathbb{Z}_{\ell}\left[\operatorname{Gal}_{\mathbb{F}_{q}}\right]}\left(T_{\ell}(A), T_{\ell}(B)\right) .
$$

If $\ell=p$, using the language of Dieudonné modules, Tate's theorem translates into the fact that the functor contravariant Dieudonné module $T_{p}(-)$ induces an isomorphism

$$
\operatorname{Hom}_{\mathbb{F}_{q}}(A, B) \otimes \mathbb{Z}_{p} \stackrel{\sim}{\longrightarrow} \operatorname{Hom}_{\mathscr{D}_{\mathbb{F}}}\left(T_{p}(B), T_{p}(A)\right),
$$

where $\mathscr{D}_{\mathbb{F}_{q}}$ is the Dieudonné ring of $\mathbb{F}_{q}$.

Remark 20. For any prime $\ell$ the $R_{w}$-linear structure on the category $\mathrm{AV}_{w}$ defined in Section 2.1 induces an enrichment of the functor $T_{\ell}(-)$ to left $R_{w} \otimes \mathbb{Z}_{\ell}$-modules for $\ell \neq p$, and to right ${ }^{1} R_{w} \otimes \mathbb{Z}_{p}$-modules for $\ell=p$.

For any $A \in \mathrm{AV}_{w}$ and any $\ell \neq p$, the action of the arithmetic Frobenius of $\mathbb{F}_{q}$ on $T_{\ell}(A)$ agrees with the action of $F_{w} \otimes 1 \in R_{w} \otimes \mathbb{Z}_{\ell}$, and we have a natural identification

$$
\operatorname{Hom}_{\mathbb{Z}_{\ell}\left[\operatorname{Gal}_{\mathbb{F}_{p}}\right]}\left(T_{\ell}(A), T_{\ell}(B)\right)=\operatorname{Hom}_{R_{w} \otimes \mathbb{Z}_{\ell}}\left(T_{\ell}(A), T_{\ell}(B)\right)
$$

for $\ell \neq p$ and all $A, B \in \mathrm{AV}_{w}$. In the special case where $q=p$, and only in this case, the Dieudonné ring $\mathscr{D}_{\mathbb{F}_{q}}$ is commutative, and hence the theory of Dieudonné

\footnotetext{
${ }^{1}$ We employ the contravariant Dieudonné theory; therefore the left $R_{w}$-module structure of the Hom-groups in $\mathrm{AV}_{w}$ turns into a right $R_{w} \otimes \mathbb{Z}_{p}$-modules structure on the corresponding Dieudonné modules. However $R_{w}$ is commutative, hence for $A$ in $\operatorname{AV}_{w}$ we can safely treat $T_{p}(A)$ as a left $R_{w} \otimes \mathbb{Z}_{p}$-module.
} 
modules of abelian varieties over the prime field $\mathbb{F}_{p}$ does not involve semilinearity aspects. For any $A \in \mathrm{AV}_{w}$ the action of $\mathscr{D}_{\mathbb{F}_{p}}$ on $T_{p}(A)$ factors through the quotient $\mathscr{D}_{\mathbb{F}_{p}} \rightarrow R_{w} \otimes \mathbb{Z}_{p}$, and Tate's theorem says that

$$
\operatorname{Hom}_{\mathscr{D}_{\mathbb{F}}}\left(T_{p}(A), T_{p}(B)\right)=\operatorname{Hom}_{R_{w} \otimes \mathbb{Z}_{p}}\left(T_{p}(B), T_{p}(A)\right)
$$

for all $A, B \in \mathrm{AV}_{w}$. So, roughly speaking, the Dieudonné theory of abelian varieties over the prime field is analogous to the theory of Tate modules at primes $\ell \neq p$, up to replacing covariance with contravariance.

For any $\pi \in W_{p}$, we choose a simple abelian variety $B_{\pi}$ over $\mathbb{F}_{p}$ whose associated Weil $p$-number represents $\pi$.

Proposition 21. Let $w \subseteq W_{p}$ be a finite set of conjugacy classes of Weil p-numbers not containing $\sqrt{p}$. There exists an abelian variety $A_{w}$ over $\mathbb{F}_{p}$ isogenous to $\prod_{\pi \in w} B_{\pi}$ such that $T_{\ell}\left(A_{w}\right)$ is free of rank 1 over $R_{w} \otimes \mathbb{Z}_{\ell}$ for all primes $\ell$. Furthermore, for any such $A_{w}$, the natural map

is an isomorphism.

$$
R_{w} \rightarrow \operatorname{End}_{\mathbb{F}_{p}}\left(A_{w}\right)
$$

Remark 22. In the case where $w$ consists of just one Weil $p$-number, the abelian variety $A_{w}$ in Proposition 21 was already considered by Waterhouse [1969, Theorem 6.1]. We observe that the product $\prod_{\pi \in w} A_{\{\pi\}}$ of the varieties constructed for each singleton $\{\pi\} \subset w$ may well fail to serve as the $A_{w}$ satisfying the properties of Proposition 21. This failure is explained by a phenomenon analogous to congruences between Weil $q$-numbers, discussed in Example 3.

Proof. Let $B$ be any abelian variety over $\mathbb{F}_{p}$ isogenous to $\prod_{\pi \in w} B_{\pi}$. For any $\pi \in W_{p}$ with $\pi \neq \sqrt{p}$, it is straightforward to verify using Honda-Tate theory [Tate 1971, Théorème 1(ii)] that

(i) all local invariants of the division ring $\operatorname{End}_{\mathbb{F}_{p}}^{0}\left(B_{\pi}\right)$ are trivial,

(ii) $[\mathbb{Q}(\pi): \mathbb{Q}]=2 \operatorname{dim}\left(B_{\pi}\right)$.

In fact, each of these conditions is equivalent to the commutativity of $\operatorname{End}_{\mathbb{F}_{p}}\left(B_{\pi}\right)$.

Since the abelian varieties $B_{\pi}, \pi \in w$, are pairwise nonisogenous, we have that $\operatorname{End}_{\mathbb{F}_{p}}(B)$ is also commutative, and isomorphic to an order of the product of CM fields $\prod_{\pi \in w} \mathbb{Q}(\pi)$. We deduce the chain of equalities

$$
\operatorname{rk}_{\mathbb{Z}}\left(\operatorname{End}_{\mathbb{F}_{p}}(B)\right)=\sum_{\pi \in w}[\mathbb{Q}(\pi): \mathbb{Q}]=\sum_{\pi \in w} 2 \operatorname{dim}\left(B_{\pi}\right)=2 \operatorname{dim}(B) .
$$

From the injectivity of the isomorphism of Theorem 19, and using the language of Dieudonné modules if $\ell=p$, it follows that the action of $R_{w} \otimes \mathbb{Q}_{\ell}=$ $\prod_{\pi \in w} \mathbb{Q}(\pi) \otimes \mathbb{Q}_{\ell}$ on

$$
V_{\ell}(B)=T_{\ell}(B) \otimes_{\mathbb{Z}_{\ell}} \mathbb{Q}_{\ell}
$$


is faithful. Hence $V_{\ell}(B)$ has rank 1 over $\prod_{\pi \in w} \mathbb{Q}(\pi) \otimes \mathbb{Q}_{\ell}$, since they both have $\operatorname{dimension} 2 \operatorname{dim}(B)$ over $\mathbb{Q}_{\ell}$ (notice that $\operatorname{dim}_{\mathbb{Q}_{p}}\left(V_{p}(B)\right)=2 \operatorname{dim}(B)$ because we work over $\mathbb{F}_{p}$ ).

Therefore, for every $\ell$ we can choose an $R_{w} \otimes \mathbb{Z}_{\ell}$-lattice

$$
\Lambda_{\ell} \subset V_{\ell}(B)
$$

which is free of rank 1 , and which contains $T_{\ell}(B)$ if $\ell \neq p$ and is contained in $T_{p}(B)$ if $\ell=p$.

If $R_{w} \otimes \mathbb{Z}_{\ell}$ is the maximal order of $\prod_{\pi \in w} \mathbb{Q}(\pi) \otimes \mathbb{Q}_{\ell}$, as occurs for almost all $\ell$, then $T_{\ell}(B)$ is necessarily free of rank 1 over $R_{w} \otimes \mathbb{Z}_{\ell}$ and we take $\Lambda_{\ell}=T_{\ell}(B)$.

Now, if $\ell \neq p$, then the finite subgroup

$$
N_{\ell}=\Lambda_{\ell} / T_{\ell}(B) \subset B\left[\ell^{\infty}\right],
$$

being an $R_{w}$-submodule, is stable under Frobenius and hence is defined over $\mathbb{F}_{p}$. The corresponding isogeny $\psi_{\ell}: B \rightarrow B / N_{\ell}$ induces an identification $\Lambda_{\ell} \simeq T_{\ell}\left(B / N_{\ell}\right)$ of $R_{w} \otimes \mathbb{Z}_{\ell}$-modules.

Similarly, the $p$-power degree isogeny $\psi_{p}: B \rightarrow B / N_{p}$, where $N_{p}$ is the $\mathbb{F}_{p^{-}}$ subgroup-scheme of $B$ corresponding to the Dieudonné module $T_{p}(B) / \Lambda_{p}$, induces an identification $T_{p}\left(B / N_{p}\right) \simeq \Lambda_{p}$ of $R_{w} \otimes \mathbb{Z}_{p}$-modules. Therefore, after applying a finite sequence of isogenies to $B$, we obtain the abelian variety $A_{w}$ with the desired property.

Lastly, by Theorem 19, the natural map

$$
R_{w} \rightarrow \operatorname{End}_{\mathbb{F}_{p}}\left(A_{w}\right)
$$

is an isomorphism after $-\otimes \mathbb{Z}_{\ell}$ for all prime numbers $\ell$, since $T_{\ell}\left(A_{w}\right) \simeq R_{w} \otimes \mathbb{Z}_{\ell}$. Therefore the last statement of the proposition follows.

Remark 23. One can show that there is a free and transitive action of the Picard group $\operatorname{Pic}\left(R_{w}\right)$ on the set of isomorphism classes of abelian varieties $A_{w}$ satisfying the conditions of Proposition 21 (see [Waterhouse 1969, Theorem 6.1.3] for the case of simple abelian varieties, i.e., $w=\{\pi\}$ ). We will discuss this below in Section 7.3.

The Gorenstein property of $R_{w}$ allows the following useful characterization of the abelian varieties $A_{w}$ satisfying the property of Proposition 21 (see also the end of $\S 4$ in [Serre and Tate 1968]).

Proposition 24. Let $w \subseteq W_{p}$ be a finite set of conjugacy classes of Weil p-numbers not containing $\sqrt{p}$, and let $A$ be an abelian variety over $\mathbb{F}_{p}$ isogenous to $\prod_{\pi \in w} B_{\pi}$. The following conditions are equivalent:

(a) $T_{\ell}(A)$ is free of rank 1 over $R_{w} \otimes \mathbb{Z}_{\ell}$, for all primes $\ell$.

(b) $\operatorname{End}_{\mathbb{F}_{p}}(A)$ is equal to the minimal central order $R_{w}$. 
Proof. Thanks to Proposition 21, we only need to show that (b) implies (a). Since $R_{w}$ is Gorenstein by Theorem 11 , its completion $R_{w} \otimes \mathbb{Z}_{\ell}$ is also Gorenstein. It follows from [Bass 1963, Theorem 6.2] that the torsion-free $R_{w} \otimes \mathbb{Z}_{\ell}$-module $T_{\ell}(A)$ is reflexive.

By (b) and Theorem 19 we have $\operatorname{End}_{R_{w} \otimes \mathbb{Z}_{\ell}}\left(T_{\ell}(A)\right)=R_{w} \otimes \mathbb{Z}_{\ell}$, so Proposition 18 yields that $T_{\ell}(A)$ is projective of rank 1 . Since $R_{w} \otimes \mathbb{Z}_{\ell}$ is a finite $\mathbb{Z}_{\ell}$-algebra, hence a product

$$
R_{w} \otimes \mathbb{Z}_{\ell}=\prod_{\lambda} R_{\lambda}
$$

of complete local rings $R_{\lambda}$, its Picard group is trivial and $T_{\ell}(A)$ is free of rank 1 as an $R_{w} \otimes \mathbb{Z}_{\ell}$-module.

We conclude the section by observing that if $A$ is an abelian variety over $\mathbb{F}_{q}$, for $q$ arbitrary, the Dieudonné module $T_{p}(A)$ has rank $2 \operatorname{dim}(A)$ over the Witt vectors $W\left(\mathbb{F}_{q}\right)$ of $\mathbb{F}_{q}$. It follows that the naive analogue of (a) can never be attained if $q>p$ for rank reasons, and the above proposition is peculiar to the $q=p$ case.

\section{Construction of the antiequivalence}

In this section we give a proof of Theorem 1. Recall from Remark 5 that for a subset $W \subseteq W_{q}$ of conjugacy classes of Weil $q$-numbers, the category $\mathrm{AV}_{W}$ is the full subcategory of $\mathrm{AV}_{q}$ consisting of all abelian varieties $A$ over $\mathbb{F}_{q}$ whose support $w(A)$ is contained in $W$.

5.1. Finite Weil support. We begin by defining the lattice $T(A)$ and its endomorphism $F$ on the increasing family of subcategories

$$
\mathrm{AV}_{w} \subseteq \mathrm{AV}_{p}^{\mathrm{com}}
$$

for finite subsets $w \subseteq W_{p}^{\text {com }}$.

Let us then assume that $\sqrt{p} \notin w$, and pick an abelian variety $A_{w}$ satisfying the condition of Proposition 21 for $w$. For any object $A$ of $\mathrm{AV}_{w}$ there is a natural $R_{w}=\operatorname{End}_{\mathbb{F}_{p}}\left(A_{w}\right)$-module structure on

$$
\mathrm{M}_{w}(A):=\operatorname{Hom}_{\mathbb{F}_{p}}\left(A, A_{w}\right) .
$$

This is the same $R_{w}$-structure described in Remark 5.

Theorem 25. Let $w \subseteq W_{p}$ be a finite set of nonreal conjugacy classes of Weil p-numbers. The functor $\mathrm{M}_{w}(-)$ induces an antiequivalence

$$
\mathrm{AV}_{w} \rightarrow \operatorname{Refl}\left(R_{w}\right)
$$

The $\mathbb{Z}$-rank of $\mathrm{M}_{w}(A)$ is $2 \operatorname{dim}(A)$. 
Proof. We begin by showing that $\mathrm{M}_{w}(-)$ is fully faithful. The map

$$
f: \operatorname{Hom}_{\mathbb{F}_{p}}\left(A^{\prime}, A^{\prime \prime}\right) \rightarrow \operatorname{Hom}_{R_{\pi}}\left(\mathrm{M}_{w}\left(A^{\prime \prime}\right), \mathrm{M}_{w}\left(A^{\prime}\right)\right)
$$

is a homomorphism of finitely generated $\mathbb{Z}$-modules, and hence it is an isomorphism if and only if it is an isomorphism after scalar extension $-\otimes \mathbb{Z}_{\ell}$ for all primes $\ell$.

We first treat the case $\ell \neq p$. For $N \in \operatorname{Refl}\left(R_{w} \otimes \mathbb{Z}_{\ell}\right)$, set

$$
N^{\vee}=\operatorname{Hom}_{R_{w} \otimes \mathbb{Z}_{\ell}}\left(N, T_{\ell}\left(A_{w}\right)\right),
$$

which is isomorphic to the $R_{w} \otimes \mathbb{Z}_{\ell}$-dual of $N$, in view of our choice of $A_{w}$. The isomorphism of Theorem 19 gives a natural isomorphism of contravariant functors

$$
\left(T_{\ell}(-)\right)^{\vee}=\operatorname{Hom}_{R_{w} \otimes \mathbb{Z}_{\ell}}\left(T_{\ell}(-), T_{\ell}\left(A_{w}\right)\right) \simeq \mathrm{M}_{w}(-) \otimes \mathbb{Z}_{\ell}
$$

on $\mathrm{AV}_{w}$ (see Remark 20). This translates into the commutative diagram

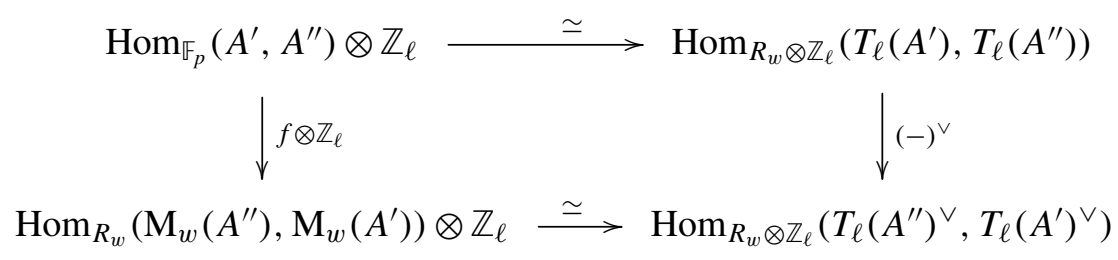

where both horizontal maps are isomorphisms as a consequence of Theorem 19. Since $R_{w} \otimes \mathbb{Z}_{\ell}$ is a completion of a Gorenstein ring by Theorem 11, it is itself Gorenstein. Because $T_{\ell}\left(A_{w}\right)$ is free of rank 1 , this implies that $N \mapsto N^{\vee}$ is an contravariant autoequivalence of $\operatorname{Refl}\left(R_{w} \otimes \mathbb{Z}_{\ell}\right)$ [Bass 1963, Theorem 6.2]. Therefore the right vertical map in the diagram is an isomorphism, and we conclude that $f \otimes \mathbb{Z}_{\ell}$ is an isomorphism as well.

Concerning the case $\ell=p$, for any $N \in \operatorname{Refl}\left(R_{w} \otimes \mathbb{Z}_{p}\right)$ we set

$$
N_{\vee}=\operatorname{Hom}_{R_{w} \otimes \mathbb{Z}_{p}}\left(T_{p}\left(A_{w}\right), N\right) .
$$

The isomorphism of Theorem 19 then gives a natural isomorphism of contravariant functors

$$
\left(T_{p}(-)\right)_{\vee}=\operatorname{Hom}_{R_{w} \otimes \mathbb{Z}_{p}}\left(T_{p}\left(A_{w}\right), T_{p}(-)\right) \simeq \mathrm{M}_{w}(-) \otimes \mathbb{Z}_{p}
$$

on $\mathrm{AV}_{w}$, which translates into the commutative diagram

$$
\begin{gathered}
\operatorname{Hom}_{\mathbb{F}_{p}}\left(A^{\prime}, A^{\prime \prime}\right) \otimes \mathbb{Z}_{p} \stackrel{\simeq}{\longrightarrow} \operatorname{Hom}_{R_{w} \otimes \mathbb{Z}_{p}}\left(T_{p}\left(A^{\prime \prime}\right), T_{p}\left(A^{\prime}\right)\right) \\
\downarrow f \otimes \mathbb{Z}_{p} \\
\operatorname{Hom}_{R_{w}}\left(\mathrm{M}_{w}\left(A^{\prime \prime}\right), \mathrm{M}_{w}\left(A^{\prime}\right)\right) \otimes \mathbb{Z}_{p} \stackrel{\simeq}{\longrightarrow} \operatorname{Hom}_{R_{w} \otimes \mathbb{Z}_{p}}\left(T_{p}\left(A^{\prime \prime}\right)_{\vee}, T_{p}\left(A^{\prime}\right)_{\vee}\right)
\end{gathered}
$$


The horizontal maps are isomorphisms by Theorem 19. Since $T_{p}\left(A_{w}\right)$ is free of rank 1 over $R_{w} \otimes \mathbb{Z}_{p}$, the right vertical map in the diagram is an isomorphism. We conclude that $f \otimes \mathbb{Z}_{p}$ is an isomorphism as well.

We have now established that the functor $A \mapsto \mathrm{M}_{w}(A)$ from $\mathrm{AV}_{w}$ to the category $\operatorname{Refl}\left(R_{w}\right)$ is fully faithful.

In order to show that $\mathrm{M}_{w}(-)$ is an equivalence, we must now show that this functor is essentially surjective. Let $M \in \operatorname{Refl}\left(R_{w}\right)$ be a reflexive module. Since $R_{w}$ is Gorenstein, the natural map $M \rightarrow \operatorname{Hom}_{R_{w}}\left(\operatorname{Hom}_{R_{w}}\left(M, R_{w}\right), R_{w}\right)$ is an isomorphism. Dualizing a presentation of the dual $\operatorname{Hom}_{R_{w}}\left(M, R_{w}\right)$ leads to a copresentation

$$
0 \longrightarrow M \longrightarrow\left(R_{w}\right)^{n} \stackrel{\psi}{\longrightarrow}\left(R_{w}\right)^{m} .
$$

Since $\mathrm{M}_{w}\left(A_{w}\right)=\operatorname{End}_{\mathbb{F}_{p}}\left(A_{w}\right)=R_{w}$, we find by full faithfulness of $\mathrm{M}_{w}(-)$ a homomorphism

$$
\Psi:\left(A_{w}\right)^{m} \rightarrow\left(A_{w}\right)^{n}
$$

with $\psi=\mathrm{M}_{w}(\Psi)$. The cokernel

$$
B=\operatorname{coker}(\Psi)
$$

exists and is an abelian variety $B \in \mathrm{AV}_{w}$. By definition of the cokernel, the functor $\mathrm{M}_{w}(-)$ is left-exact; hence

$$
0 \longrightarrow \mathrm{M}_{w}(B) \longrightarrow \mathrm{M}_{w}\left(\left(A_{w}\right)^{n}\right) \stackrel{\mathrm{M}_{w}(\Psi)}{\longrightarrow} \mathrm{M}_{w}\left(\left(A_{w}\right)^{m}\right),
$$

and so

$$
M \simeq \mathrm{M}_{w}(B)
$$

as $R_{w}$-modules. This completes the proof of essential surjectivity.

We are only left with showing that $\operatorname{rk}_{\mathbb{Z}}\left(\operatorname{Hom}_{\mathbb{F}_{p}}\left(A, A_{w}\right)\right)=2 \operatorname{dim}(A)$ for all $A$ in $\mathrm{AV}_{w}$. The statement is additive in $A$ and depends only on the isogeny class of $A$ and $A_{w}$. Recall that for any $\pi \in W_{p}$ we have chosen a simple abelian variety $B_{\pi}$ over $\mathbb{F}_{p}$ whose associated Weil $p$-number represents $\pi$. Because $A_{w}$ is isogenous to $\prod_{\pi \in w} B_{\pi}$, it is enough to show that for any $\pi \in w$ we have

$$
\operatorname{rk}_{\mathbb{Z}}\left(\operatorname{Hom}_{\mathbb{F}_{p}}\left(B_{\pi}, \prod_{\pi^{\prime} \in w} B_{\pi^{\prime}}\right)\right)=2 \operatorname{dim}\left(B_{\pi}\right) .
$$

This follows from the equality $\operatorname{rk}_{\mathbb{Z}}\left(\operatorname{End}_{\mathbb{F}_{p}}\left(B_{\pi}\right)\right)=2 \operatorname{dim}\left(B_{\pi}\right)$ for all Weil $p$-numbers $\pi \nsim \sqrt{p}$ [Tate 1971, Théorème 1(ii)], and the proof of the theorem is complete.

5.2. The direct system. In order to prove Theorem 1 , we construct a direct system $\mathscr{A}=\underline{\lim } A_{w}$ consisting of abelian varieties $A_{w}$ indexed by finite sets $w$ of Weil $p$-numbers not containing $\sqrt{p}$, and having the property stated in Proposition 21. 
Let $v \subseteq w$ be two finite sets of nonreal Weil $p$-numbers. By means of the canonical surjection

$$
\operatorname{pr}_{v, w}: R_{w} \rightarrow R_{v},
$$

we may consider $R_{v}$-modules as $R_{w}$-modules such that the action factors over $\mathrm{pr}_{v, w}$. Lemma 13 shows that

$$
\operatorname{Refl}\left(R_{v}\right) \subseteq \operatorname{Refl}\left(R_{w}\right)
$$

is a full subcategory. After choosing abelian varieties $A_{v}$ and $A_{w}$ as in Proposition 21, associated to the sets $v$ and $w$ respectively, we obtain a diagram of functors

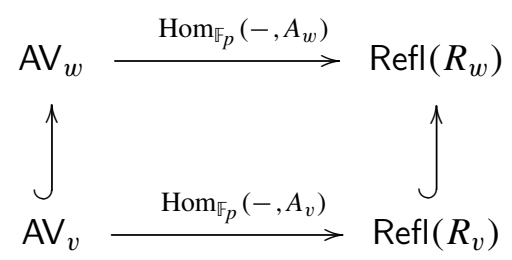

where the vertical functors are natural full subcategories. This diagram need not commute for arbitrary unrelated choices $A_{w}$ and $A_{v}$. The next proposition shows that for every $A_{w}$ there is a canonical abelian subvariety $A_{v, w} \subseteq A_{w}$ that leads to a choice of $A_{v}$ for which (5-3) commutes.

Proposition 26. Let $w$ be a set of nonreal conjugacy classes of Weil p-numbers, let $A_{w}$ be an abelian variety over $\mathbb{F}_{p}$ such that $\operatorname{End}_{\mathbb{F}_{p}}\left(A_{w}\right)=R_{w}$, and let $v \subseteq w$ be any subset. Then the subgroup generated by all images

$$
A_{v, w}:=\left\langle\operatorname{im}(f): f: B \rightarrow A_{w}, B \in \mathrm{A \bigvee}_{v}\right\rangle \subseteq A_{w}
$$

satisfies the following:

(1) $A_{v, w}$ belongs to $\mathrm{AV}_{v}$ and is an abelian subvariety of $A_{w}$.

(2) $T_{\ell}\left(A_{v, w}\right)$ is free of rank 1 over $R_{v} \otimes \mathbb{Z}_{\ell}$ for all primes $\ell$.

(3) The diagram (5-3) commutes when $A_{w}$ is chosen to be the abelian variety associated to $w$ and $A_{v}=A_{v, w}$ as that associated to $v$.

(4) The abelian variety $A_{v, w}$ is the image of any map $f: B \rightarrow A_{w}$ such that $w(B)=v$ and $w(\operatorname{coker}(f))=w \backslash v$.

Proof. Assertion (1) is obvious and assertion (3) follows from the natural equality

$$
\operatorname{Hom}_{\mathbb{F}_{p}}\left(B, A_{v, w}\right)=\operatorname{Hom}_{\mathbb{F}_{p}}\left(B, A_{w}\right)
$$

for every $B \in \mathrm{AV}_{v}$, since every morphism $f: B \rightarrow A_{w}$ takes values in the subvariety $A_{v, w} \subseteq A_{w}$. 
Assertion (4) is obvious once we pass to the semisimple category of abelian varieties up to isogeny. Therefore $f(B)$ and $A_{v, w}$ have the same dimension. Since by definition $f(B) \subseteq A_{v, w}$, we obtain the claimed equality.

It remains to verify assertion (2), which by Proposition 24 is equivalent to $\operatorname{End}_{\mathbb{F}_{p}}\left(A_{v, w}\right)=R_{v}$. The natural map

$$
R_{w}=\operatorname{End}_{\mathbb{F}_{p}}\left(A_{w}\right)=\mathrm{M}_{w}\left(A_{w}\right) \rightarrow \mathrm{M}_{w}\left(A_{v, w}\right)=\operatorname{End}_{\mathbb{F}_{p}}\left(A_{v, w}\right)
$$

factors through the quotient map $\mathrm{pr}_{v, w}: R_{w} \rightarrow R_{v}$. In order to prove (2), it is enough to show that (5-4) is surjective. It suffices to verify surjectivity after $-\otimes \mathbb{Z}_{\ell}$ for every prime number $\ell$.

Assume first that $\ell \neq p$. Let $C$ be the quotient abelian variety $C=A_{w} / A_{v, w}$. There is an exact sequence of reflexive $R_{w} \otimes \mathbb{Z}_{\ell}$-modules

$$
0 \longrightarrow \mathrm{T}_{\ell}\left(A_{v, w}\right) \longrightarrow \mathrm{T}_{\ell}\left(A_{w}\right) \longrightarrow \mathrm{T}_{\ell}(C) \longrightarrow 0,
$$

and its Ext-sequence contains

$$
\begin{aligned}
\operatorname{Hom}_{R_{w} \otimes \mathbb{Z}_{\ell}}\left(\mathrm{T}_{\ell}\left(A_{w}\right), \mathrm{T}_{\ell}\left(A_{w}\right)\right) \longrightarrow & \operatorname{Hom}_{R_{w} \otimes \mathbb{Z}_{\ell}}\left(\mathrm{T}_{\ell}\left(A_{v, w}\right), \mathrm{T}_{\ell}\left(A_{w}\right)\right) \\
& \longrightarrow \operatorname{Ext}_{R_{w} \otimes \mathbb{Z}_{\ell}}^{1}\left(\mathrm{~T}_{\ell}(C), \mathrm{T}_{\ell}\left(A_{w}\right)\right) .
\end{aligned}
$$

The $\mathrm{Ext}^{1}$-term vanishes by Lemma 17 . Thus Theorem 19 shows the surjectivity of

$$
\begin{aligned}
\mathrm{M}_{w}\left(A_{w}\right) \otimes \mathbb{Z}_{\ell}=\operatorname{Hom}_{R_{w} \otimes \mathbb{Z}_{\ell}} & \left.\mathrm{T}_{\ell}\left(A_{w}\right), \mathrm{T}_{\ell}\left(A_{w}\right)\right) \\
& \rightarrow \operatorname{Hom}_{R_{w} \otimes \mathbb{Z}_{\ell}}\left(\mathrm{T}_{\ell}\left(A_{v, w}\right), \mathrm{T}_{\ell}\left(A_{w}\right)\right)=\mathrm{M}_{w}\left(A_{v, w}\right) \otimes \mathbb{Z}_{\ell} .
\end{aligned}
$$

If $\ell=p$, then the inclusion $A_{v, w} \subseteq A_{w}$ gives a surjection of reflexive $R_{w} \otimes \mathbb{Z}_{p^{-}}$ modules

$$
\mathrm{T}_{p}\left(A_{w}\right) \rightarrow \mathrm{T}_{p}\left(A_{v, w}\right) .
$$

Since $T_{p}\left(A_{w}\right)$ is free over $R_{w} \otimes \mathbb{Z}_{p}$, we obtain a surjection

$$
\operatorname{Hom}_{R_{w} \otimes \mathbb{Z}_{p}}\left(T_{p}\left(A_{w}\right), T_{p}\left(A_{w}\right)\right) \rightarrow \operatorname{Hom}_{R_{w} \otimes \mathbb{Z}_{p}}\left(T_{p}\left(A_{w}\right), T_{p}\left(A_{v, w}\right)\right),
$$

which, by Theorem 19 , says that $\mathbf{M}_{w}\left(A_{w}\right) \otimes \mathbb{Z}_{p} \rightarrow \mathbf{M}_{w}\left(A_{v, w}\right) \otimes \mathbb{Z}_{p}$ is surjective. This completes the proof of the proposition.

5.3. Proof of the main result. We are now ready to prove our main result. We must show that the abelian varieties $A_{w}$ that exist by Proposition 21 for each $w$, and which yield equivalences of the desired type on the respective full subcategories $\mathrm{AV}_{w}$ by Theorem 25, can be chosen in a compatible way for every $v \subseteq w$. This requires a two-step process. We use the notation of Proposition 26.

- First, we establish compatibility on the set-theoretic level: we must fix isomorphism classes for each $A_{w}$ such that $A_{v} \simeq A_{v, w}$ for every $v \subseteq w$. 
- Secondly, we categorize the first choice: we must choose isomorphisms $A_{v} \simeq A_{v, w}$ such that the inclusions $\varphi_{w, v}: A_{v} \simeq A_{v, w} \subseteq A_{w}$ obey the cocycle condition $\varphi_{w, v} \circ \varphi_{v, u}=\varphi_{w, u}$ for $u \subseteq v \subseteq w$, and thus construct an ind-system $\mathscr{A}=\left(A_{w}, \varphi_{w, v}\right)$.

Proof of Theorem 1. For any finite set $w \subseteq W_{p}$ that avoids $\sqrt{p}$, let $Z(w)$ be the set of isomorphism classes $[A]$ of abelian varieties $A$ in $\mathrm{AV}_{w}$ such that the natural map $R_{w} \rightarrow \operatorname{End}_{\mathbb{F}_{p}}(A)$ is an isomorphism. The elements of $Z(w)$ all belong to the same isogeny class, and so $Z(w)$ is finite, since there are only finitely many isomorphism classes of abelian varieties over a finite field lying in a given isogeny class (in fact, finiteness holds for isomorphism classes of abelian varieties of fixed dimension [Milne 1986, Corollary 18.9]). Moreover, the set $Z(w)$ is nonempty by Proposition 21.

For any pair $v \subseteq w$ of finite sets of nonreal Weil $p$-numbers, we construct a map

$$
\zeta_{v, w}: Z(w) \rightarrow Z(v)
$$

given by $\zeta_{v, w}([A])=[B]$, where $B$ is the abelian subvariety of $A$ generated by the image of all $f: C \rightarrow A$ with $w(C) \subseteq v$. Proposition 26 states that $\zeta_{v, w}$ indeed takes values in $Z(v)$.

These maps satisfy the compatibility condition

$$
\zeta_{u, w}=\zeta_{u, v} \zeta_{v, w}
$$

for all tuples $u \subseteq v \subseteq w$, hence they define a projective system

$$
\left(Z(w), \zeta_{v, w}\right)
$$

indexed by finite subsets $w \subseteq W_{p}$ with $\sqrt{p} \notin w$. Since the sets $Z(w)$ are finite and nonempty, a standard compactness argument shows that the inverse limit is not empty:

$$
Z=\lim _{w} Z(w) \neq \varnothing
$$

We choose a compatible ${ }^{2}$ system $z=\left(z_{w}\right) \in Z$ of isomorphism classes of abelian varieties.

Now we would like to choose abelian varieties $A_{w}$ in each class $z_{w}$, and inclusions

$$
\varphi_{w, v}: A_{v} \rightarrow A_{w}
$$

\footnotetext{
${ }^{2}$ We will see later in Remark 40 that $\zeta_{v, w}$ is always surjective. This extra piece of information simplifies the construction of the system marginally. However, we find it conceptually easier to deduce this fact from the antiequivalence of Theorem 1, hence the order of the assertions and proofs.
} 
for every $v \subseteq w$ that are isomorphic to the inclusion from Proposition 26 in a compatible way: for $u \subseteq v \subseteq w$ we want

$$
\varphi_{w, u}=\varphi_{w, v} \varphi_{v, u} .
$$

Because the set of Weil numbers is countable, we may choose a cofinal totally ordered subsystem of finite subsets of $W_{p}^{\text {com }}$

$$
w_{1} \subseteq w_{2} \subseteq \cdots \subseteq w_{i} \subseteq \cdots
$$

Working first with this totally ordered subsystem, we can construct a direct system

$$
\mathscr{A}_{0}=\left(A_{w_{i}}, \varphi_{w_{j}, w_{i}}\right)
$$

of abelian varieties, as desired, by induction. If $A_{w_{i}}$ is already constructed, then we choose $A_{w_{i+1}}$ in $z_{w_{i+1}}$ and deduce from $\zeta_{w_{i}, w_{i+1}}\left(z_{w_{i+1}}\right)=z_{w_{i}}$ that there is an inclusion $\varphi_{w_{i+1}, w_{i}}: A_{w_{i}} \rightarrow A_{w_{i+1}}$ as desired.

Once this is achieved, we may identify all transfer maps of the restricted system $\mathscr{A}_{0}$ with inclusions. Now we can extend the directed system $\mathscr{A}_{0}$ from the index set $\left\{w_{i}: i \in \mathbb{N}\right\}$ to an ind-object $\mathscr{A}$ on all finite subsets of $W_{p}$. For a general finite $w \subset W_{p}$ we choose $i$ large enough such that $w \subseteq w_{i}$, and define

$$
A_{w}:=A_{w, w_{i}} \subseteq A_{w_{i}}
$$

by means of the construction of Proposition 26. This choice is well defined, i.e., independent of $i \gg 0$. Furthermore, there are compatible transfer maps $\varphi_{v, w}: A_{v} \rightarrow$ $A_{w}$ for all $v \subseteq w$ that lead to the desired direct system

$$
\mathscr{A}=\left(A_{w}, \varphi_{w, v}\right) .
$$

In the sense of ind-objects we have $\mathscr{A}_{0} \simeq \mathscr{A}$ and so $\mathscr{A}_{0}$ would suffice for Theorem 1 , but we wanted to restore symmetry and have $A_{w}$ for all finite subsets $w \subseteq W_{p}^{\text {com }}$.

Let $A$ be any element of $\mathrm{AV}_{p}^{\text {com }}$, and set

$$
T(A)=\operatorname{Hom}_{\mathbb{F}_{p}}(A, \mathscr{A})=\underset{w}{\lim } \operatorname{Hom}_{\mathbb{F}_{p}}\left(A, A_{w}\right)=\underset{w}{\lim } \mathrm{M}_{w}(A) .
$$

The groups $\operatorname{Hom}_{\mathbb{F}_{p}}\left(A, A_{w}\right)$ are stable when $w$ is large enough. More precisely, if $w, w^{\prime}$ are finite sets of Weil $p$-numbers with $w(A) \subseteq w \subseteq w^{\prime}$, then the map

$$
\varphi_{w^{\prime}, w} \circ-: \operatorname{Hom}_{\mathbb{F}_{p}}\left(A, A_{w}\right) \rightarrow \operatorname{Hom}_{\mathbb{F}_{p}}\left(A, A_{w^{\prime}}\right)
$$

is an isomorphism (see Proposition 26). Moreover, $T(-)$ restricted to $\mathrm{AV}_{w}$ recovers the functor $\mathrm{M}_{w}(-)$ of Theorem 25 constructed using the object $A_{w}$ of $\mathscr{A}$, and induces an antiequivalence between $\mathrm{AV}_{w}$ and $\operatorname{Refl}\left(R_{w}\right)$. 
Observe that, by the naturality of the Frobenius isogeny, for any finite $w \subseteq W_{p}$ avoiding $\sqrt{p}$ and any $f \in \operatorname{Hom}_{\mathbb{F}_{p}}\left(A, A_{w}\right)$ the diagram

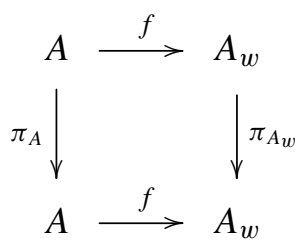

is commutative. This implies that, for $w$ sufficiently large, the action of $F_{w} \in R_{w}$ on $T(A)$ is given by $T\left(\pi_{A}\right)$, the morphism induced by the Frobenius isogeny $\pi_{A}$ via functoriality of $T$.

Compatibility in $w$ shows that $T(-)$ induces an antiequivalence

$$
T=\underline{\lim _{\longrightarrow}} M_{w}: \mathrm{AV}_{p}^{\mathrm{com}}=\underline{\lim _{w}} \mathrm{AV}_{w} \stackrel{\sim}{\longrightarrow} \underset{w}{\lim } \operatorname{Refl}\left(R_{w}\right)=\operatorname{Refl}\left(\mathscr{R}_{p}^{\mathrm{com}}\right) .
$$

Due to the remarks of Section 3.2, this is precisely the claim of Theorem 1, and so its proof is complete.

\section{Properties of the functor $T$}

6.1. Recovering Tate and Dieudonné module. Let $A$ be an abelian variety over $\mathbb{F}_{p}$, and set $w=w(A)$. We explain here how the $R_{w} \otimes \mathbb{Z}_{\ell}$-modules $T_{\ell}(A)$ can be recovered from the pair $(T(A), F)$ attached to $A$ by Theorem 1 . We set

$$
\mathscr{R}_{\ell}=\underset{w}{\lim }\left(R_{w} \otimes \mathbb{Z}_{\ell}\right)
$$

for all prime numbers $\ell$, where in the projective limit $w$ ranges through all finite subsets of $W_{p}^{\text {com }}$, and define

$$
T_{\ell}(\mathscr{A})= \begin{cases}{\underset{\lim }{\longrightarrow}}_{w} T_{\ell}\left(A_{w}\right) & \ell \neq p, \\ \varliminf_{w} T_{p}\left(A_{w}\right) & \ell=p,\end{cases}
$$

as the direct limit if $\ell \neq p$ and the projective limit if $\ell=p$ of the system obtained by applying $T_{\ell}(-)$ to the direct system $\mathscr{A}=\left(A_{w}\right)_{w}$ constructed in the proof of Theorem 1.

We first discuss the $\ell$-adic Tate module, and assume $\ell \neq p$. Since for $v \subseteq w$ the map $A_{v} \rightarrow A_{w}$ is an inclusion of abelian varieties, the induced map $T_{\ell}\left(A_{v}\right) \rightarrow$ $T_{\ell}\left(A_{w}\right)$ is the inclusion of a direct summand, at least as $\mathbb{Z}_{\ell}$-modules. Hence $T_{\ell}(\mathscr{A})$ is a free $\mathbb{Z}_{\ell}$-module of countable infinite rank.

Proposition 27. Let $A$ be an abelian variety over $\mathbb{F}_{p}$ with $\sqrt{p} \notin w(A)$. There is a natural isomorphism of $\mathscr{R}_{\ell}$-modules

$$
T_{\ell}(A) \stackrel{\sim}{\rightarrow} \operatorname{Hom}_{\mathscr{R}_{\ell}}\left(T(A) \otimes \mathbb{Z}_{\ell}, T_{\ell}(\mathscr{A})\right) .
$$


Proof. Let $w \subseteq W_{p}^{\text {com }}$ be a finite set containing $w(A)$. Since $R_{w} \otimes \mathbb{Z}_{\ell}$ is Gorenstein, dualizing (5-1) yields the first equality in

$$
T_{\ell}(A)=\operatorname{Hom}_{R_{w} \otimes \mathbb{Z}_{\ell}}\left(M_{w}(A) \otimes \mathbb{Z}_{\ell}, T_{\ell}\left(A_{w}\right)\right)=\operatorname{Hom}_{\mathscr{R}_{\ell}}\left(T(A) \otimes \mathbb{Z}_{\ell}, T_{\ell}(\mathscr{A})\right) .
$$

The second equality holds, because $T_{\ell}\left(A_{w}\right) \subseteq T_{\ell}(\mathscr{A})$ is the maximal submodule on which $\mathscr{R}_{\ell}$ acts through its quotient $\mathscr{R}_{\ell} \rightarrow R_{w} \otimes \mathbb{Z}_{\ell}$.

Now we address the contravariant Dieudonné module $T_{p}(A)$. We endow $T_{p}(\mathscr{A})$ with the projective limit topology. If $M$ is a topological $\mathscr{R}_{p}$-module which is finite and free over $\mathbb{Z}_{p}$, then the action of $\mathscr{R}_{p}$ on $M$ factors through $\mathscr{R}_{p} \rightarrow R_{w} \otimes \mathbb{Z}_{p}$ for some large enough $w$, by compactness of $M$. We denote by

$$
M \widehat{\otimes}_{\mathscr{R}_{p}} T_{p}(\mathscr{A})=\lim _{w \gg \varnothing} M \otimes_{R_{w} \otimes \mathbb{Z}_{p}} T_{p}\left(A_{w}\right)
$$

the continuous tensor product.

Proposition 28. Let $A$ be an abelian variety over $\mathbb{F}_{p}$ with $\sqrt{p} \notin w(A)$. There is a natural isomorphism of $\mathscr{R}_{p}$-modules

$$
T_{p}(A)=\left(T(A) \otimes \mathbb{Z}_{p}\right) \widehat{\otimes}_{\mathscr{R}_{p}} T_{p}(\mathscr{A}) .
$$

Proof. Let $w \subseteq W_{p}^{\text {com }}$ be a finite set containing $w(A)$. We deduce from (5-2) a natural identification

$$
\begin{aligned}
T_{p}(A) & =\operatorname{Hom}_{R_{w} \otimes \mathbb{Z}_{p}}\left(T_{p}\left(A_{w}\right), T_{p}(A)\right) \otimes_{R_{w} \otimes \mathbb{Z}_{p}} T_{p}\left(A_{w}\right) \\
& =\mathrm{M}_{w}(A) \otimes_{R_{w}} T_{p}\left(A_{w}\right)=\left(T(A) \otimes \mathbb{Z}_{p}\right) \widehat{\otimes}_{\mathscr{R}_{p}} T_{p}(\mathscr{A}),
\end{aligned}
$$

because for $w(A) \subseteq w \subseteq w^{\prime}$ the natural maps

$$
\left(T(A) \otimes \mathbb{Z}_{p}\right) \otimes_{R_{w^{\prime}} \otimes \mathbb{Z}_{p}} T_{p}\left(A_{w^{\prime}}\right) \rightarrow\left(T(A) \otimes \mathbb{Z}_{p}\right) \otimes_{R_{w} \otimes \mathbb{Z}_{p}} T_{p}\left(A_{w}\right)
$$

are isomorphisms.

6.2. Isogenies and inclusions. We discuss how the functor $T(-)$ detects isogenies and inclusions.

Proposition 29. Let $A$ and $B$ be abelian varieties in $\mathrm{AV}_{p}^{\text {com }}$.

(1) The map $f: B \rightarrow A$ is an isogeny if and only if $T(f) \otimes \mathbb{Q}$ is an isomorphism.

(2) For an isogeny $f: B \rightarrow A$, the map $T(f)$ is injective and the image is of index

$$
\operatorname{deg}(f)=|\operatorname{coker}(T(f))| .
$$

Proof. (1) An isogeny $f$ has an inverse up to multiplication-by- $n$ map for $n=\operatorname{deg}(f)$. Therefore $T(f)$ is an isomorphism after inverting $\operatorname{deg}(f)$.

Conversely, if $f$ is not an isogeny, then either $\operatorname{ker}(f)$ or coker $(f)$ have a nontrivial abelian variety as a direct summand up to isogeny. In the presence of such a direct summand the map $T(f) \otimes \mathbb{Q}$ cannot be an isomorphism. 
(2) We indicate the $\ell$-primary part by an index $\ell$. Then using Proposition 27 , for $\ell \neq p$ we have

$|\operatorname{coker}(T(f))|_{\ell}=\left|\operatorname{coker}(T(f)) \otimes \mathbb{Z}_{\ell}\right|=\left|\operatorname{coker}\left(T_{\ell}(f)^{\vee}: T_{\ell}(A)^{\vee} \rightarrow T_{\ell}(B)^{\vee}\right)\right|$.

The duals here are $\operatorname{Hom}\left(-, R_{w} \otimes \mathbb{Z}_{\ell}\right)$. Since $R_{w} \otimes \mathbb{Z}_{\ell}$ is reduced Gorenstein of dimension 1, we can use [Bass 1963, Theorem 6.3(4)] and induction on the length to see that

$\left|\operatorname{coker}\left(T_{\ell}(f)^{\vee}: T_{\ell}(A)^{\vee} \rightarrow T_{\ell}(B)^{\vee}\right)\right|=\left|\operatorname{coker}\left(T_{\ell}(f): T_{\ell}(B) \rightarrow T_{\ell}(A)\right)\right|=|\operatorname{ker}(f)|_{\ell}$. If $\ell=p$, using Proposition 28 yields

$$
\begin{aligned}
|\operatorname{coker}(T(f))|_{p}=\left|\operatorname{coker}(T(f)) \otimes \mathbb{Z}_{p}\right| & =\left|\operatorname{coker}\left(T_{p}(f): T_{p}(A) \rightarrow T_{p}(B)\right)\right| \\
& =|\operatorname{ker}(f)|_{p},
\end{aligned}
$$

where the last equality follows from Dieudonné theory.

Proposition 30. Let $A$ and $B$ be abelian varieties in $\mathrm{AV}_{p}^{\text {com }}$. For a map $f: B \rightarrow A$, the following are equivalent:

(a) $T(f): T(A) \rightarrow T(B)$ is surjective.

(b) The map $f$ can be identified with the inclusion of an abelian subvariety.

Proof. If $T(f)$ is surjective, Proposition 27 shows that the induced map $T_{\ell}(B) \rightarrow$ $T_{\ell}(A)$ is injective. Therefore $\operatorname{ker}(f)$ is at most a finite group scheme. We may therefore replace $A$ by the image $A_{0}$ of $B \rightarrow A$ and thus reduce to the case of the isogeny $f_{0}: B \rightarrow A_{0}$. Here Proposition 29 implies that $\operatorname{deg}\left(f_{0}\right)=1$, hence $B=A_{0}$ and $f$ is indeed an inclusion of an abelian subvariety.

Conversely, if $f: B \rightarrow A$ is an inclusion, then there is a map $g: A \rightarrow B$ such that $g f: B \rightarrow B$ is an isogeny. Therefore $T(f)$ has at least an image of finite index. The image of $T(f)$ is a reflexive submodule in the image of the equivalence $T(-)$, so that there is an abelian variety $C$ and a factorization $B \rightarrow C \rightarrow A$ with $T(A) \rightarrow T(C)$ surjective and $T(C) \subseteq T(B)$ an inclusion.

We have already proven that $C \rightarrow A$ is an abelian subvariety, and it is easy to see that $B \rightarrow C$ is an isogeny. Therefore $B \rightarrow C$ is an isomorphism.

As an application, we prove a variant for objects of $\mathrm{AV}_{p}$ of Waterhouse's theorem on possible endomorphism rings of $\mathbb{F}_{p}$-simple abelian varieties over $\mathbb{F}_{p}$; see [Waterhouse 1969, Theorem 6.1.2]:

Theorem 31. Let $w$ be a set of conjugacy classes of nonreal Weil p-numbers. Then the following are equivalent:

(a) $S$ is an order in $R_{w} \otimes \mathbb{Q}$ containing $R_{w}$.

(b) $S$ is isomorphic as an $R_{w}$-algebra to $\operatorname{End}_{\mathbb{F}_{p}}(B)$ for an abelian variety $B$ with $w(B)=w$ whose simple factors up to isogeny occur with multiplicity 1 . 
Proof. Since $R_{w}$ is the minimal central order for abelian varieties $B$ with $w(B)=w$, it is clear that (b) implies (a).

Conversely, if $S$ is an order containing $R_{w}$, then $S$ is a reflexive $R_{w}$-module and thus corresponds to an abelian variety $B$. Let $A_{w}$ be the abelian variety occurring in the ind-system pro-representing $T(-)$, so that $T\left(A_{w}\right)=R_{w}$. The inclusion $R_{w} \subseteq S$ corresponds to an isogeny $\varphi: B \rightarrow A_{w}$ by Proposition 29, so that $B$ has the required Weil support and product structure up to isogeny. Moreover,

$$
\operatorname{End}_{\mathbb{F}_{p}}(B)=\operatorname{End}_{R_{w}}(S)=\left\{\lambda \in R_{w} \otimes \mathbb{Q}: \lambda S \subseteq S\right\}=S
$$

shows (a) implies (b).

\section{Ambiguity and comparison}

The construction of the functor $T(-)$ in Section 5.3 depends on the choice of an ind-abelian variety $\mathscr{A}$. For the sake of distinguishing the different choices, we set in this section

$$
T_{\mathscr{A}}(-)=\operatorname{Hom}_{\mathbb{F}_{p}}(-, \mathscr{A}) .
$$

\subsection{Continuous line bundles.}

Definition 32. Let $W \subseteq W_{q}$ be a subset. Let us denote by $\mathscr{R}_{W}$ the pro-ring $\left(R_{w}\right)$, where $w$ ranges over the finite subsets of $W$.

(1) An $\mathscr{R}_{W}$-module is a pro-system $\mathcal{M}=\left(M_{w}\right)$ with $w$ ranging over the finite subsets of $W$, such that $M_{w}$ is an $R_{w}$-module and the maps $M_{w} \rightarrow M_{v}$ for $v \subseteq w$ are $R_{w}$-module homomorphisms (where $R_{w}$ acts on $M_{v}$ via the projection $R_{w} \rightarrow R_{v}$ ). Homomorphisms of $\mathcal{M}$ are levelwise $R_{w}$-module homomorphisms.

(2) An $\mathscr{R}_{W}$-module $\mathcal{M}$ is invertible if for all $w \subseteq W$ the $R_{w}$-module $M_{w}$ is invertible and for $v \subseteq w$ the maps $M_{w} \rightarrow M_{v}$ are surjective (equivalently, they induce a natural isomorphism $M_{w} \otimes_{R_{w}} R_{v} \simeq M_{v}$ ).

(3) The set of isomorphism classes of invertible $\mathscr{R}_{W}$-modules forms a group, denoted by $\operatorname{Pic}\left(\mathscr{R}_{W}\right)$, under levelwise tensor products, the Picard group of $\mathscr{R}_{W}$.

For a finite set $w$ of conjugacy classes of Weil $q$-numbers, we set $X_{w}=\operatorname{Spec}\left(R_{w}\right)$ and consider the ind-schemes

$$
\mathscr{X}=\underset{w}{\lim } X_{w},
$$

and for a subset $W \subseteq W_{q}$ the ind-scheme

$$
\mathscr{X}_{W}=\underset{w \subseteq W}{\lim _{w \subseteq}} X_{w},
$$


with closed immersions as transfer maps, all denoted $i$, induced by the projections $\operatorname{pr}_{v, w}: R_{w} \rightarrow R_{v}$. The invertible $\mathscr{R}_{W}$-modules are just line bundles on $\mathscr{X}_{W}$, and

$$
\operatorname{Pic}\left(\mathscr{R}_{W}\right)=\operatorname{Pic}\left(\mathscr{X}_{W}\right)=\mathrm{H}^{1}\left(\mathscr{X}_{W}, \mathscr{O}^{\times}\right) .
$$

Since $\mathcal{O}_{\mathscr{X}_{W}}^{\times}=\varliminf_{w \subseteq W} i_{*} \mathcal{O}_{X_{w}}^{\times}$, we find an exact sequence

$$
0 \longrightarrow \lim _{w \subseteq W}^{1} R_{w}^{\times} \longrightarrow \operatorname{Pic}\left(\mathscr{R}_{W}\right) \longrightarrow \lim _{w \subseteq W} \operatorname{Pic}\left(R_{w}\right) \longrightarrow 0 .
$$

The quotient of $\operatorname{Pic}\left(\mathscr{R}_{W}\right)$ given by $\lim _{w} \operatorname{Pic}\left(R_{w}\right)$ parametrizes the choices of a compatible system of isomorphism classes of rank-1 $R_{w}$-modules $M_{w}$. The $\varlimsup^{1}{ }^{1}$ term parametrizes all choices of transfer maps to obtain an invertible $\mathscr{R}_{W}$-module $\mathcal{M}=\left(M_{w}\right)$ from a given compatible choice of isomorphism classes of invertible $R_{w}$-modules at every level.

Proposition 33. Let $V \subseteq W \subseteq W_{q}$ be subsets. Then the natural restriction map

$$
\operatorname{Pic}\left(\mathscr{R}_{W}\right) \rightarrow \operatorname{Pic}\left(\mathscr{R}_{V}\right)
$$

is surjective.

Proof. For $v \subseteq w$, define Zariski sheaves $\mathscr{K}_{v, w}$ on $\mathscr{X}_{W}$ by the short exact sequence

$$
0 \longrightarrow \mathscr{K}_{v, w} \longrightarrow i_{*} \mathrm{O}_{X_{w}}^{\times} \longrightarrow i_{*} \mathrm{O}_{X_{v}}^{\times} \longrightarrow 0 .
$$

Then $\mathscr{K}_{V, W}=\lim _{w \subseteq W} \mathscr{K}_{w \cap V, w}$ is the kernel of $\mathcal{O}_{\mathscr{X}_{W}}^{\times} \rightarrow i_{*} \mathcal{O}_{\mathscr{X}_{V}}^{\times}$. The Zariski cohomology sequence yields an exact sequence

$$
\operatorname{Pic}\left(\mathscr{R}_{W}\right) \longrightarrow \operatorname{Pic}\left(\mathscr{R}_{V}\right) \longrightarrow \mathrm{H}^{2}\left(\mathscr{X}_{W}, \mathscr{K}_{V, W}\right),
$$

and it remains to show vanishing of $\mathrm{H}^{2}\left(\mathscr{X}_{W}, \mathscr{K}_{V, W}\right)$. The pro-structure of $\mathscr{K}_{V, W}$ leads to a short exact sequence

$0 \longrightarrow \lim _{w \subseteq W} \mathrm{H}^{1}\left(X_{w}, \mathscr{K}_{w \cap V, w}\right) \longrightarrow \mathrm{H}^{2}\left(\mathscr{X}_{W}, \mathscr{K}_{V, W}\right) \longrightarrow \lim _{w \subseteq W} \mathrm{H}^{2}\left(X_{w}, \mathscr{K}_{w \cap V, w}\right) \longrightarrow 0$

The lim-term on the right vanishes by cohomological dimension because $\operatorname{dim}\left(X_{w}\right)$ is 1 . The $\varliminf^{1}$-term on the left vanishes, as we claim that $\left(\mathrm{H}^{1}\left(X_{w}, \mathscr{K}_{w \cap V, w}\right)\right)_{w \subseteq W}$ is a surjective system, and hence a Mittag-Leffler system. Indeed, for finite subsets $w \subseteq w^{\prime} \subseteq W$, the cokernel $\mathscr{C}_{w, w^{\prime}}$ of

$$
\mathscr{K}_{w^{\prime} \cap V, w^{\prime}} \rightarrow \mathscr{K}_{w \cap V, w}
$$

is a sheaf with support in at most the finitely many points of $X_{w^{\prime}}$ that are contained in more than one irreducible component, and so $\mathrm{H}^{1}\left(X_{w^{\prime}}, \mathscr{C}_{w}, w^{\prime}\right)=0$. Since $\mathrm{H}^{1}\left(X_{w^{\prime}},-\right)$ is right exact, we have an exact sequence

$$
\mathrm{H}^{1}\left(X_{w^{\prime}}, \mathscr{K}_{w^{\prime} \cap V, w^{\prime}}\right) \rightarrow \mathrm{H}^{1}\left(X_{w}, \mathscr{K}_{w \cap V, w}\right) \rightarrow \mathrm{H}^{1}\left(X_{w^{\prime}}, \mathscr{C}_{w, w^{\prime}}\right)=0,
$$

from which we deduce the claim. 
7.2. Mixed tensor products. We recall Serre and Tate's well-known tensor product construction (see [Giraud 1968] for the parallel Hom-construction explaining a construction of Shimura and Taniyama). Let $A$ be an abelian variety over $\mathbb{F}_{q}$ and $M$ a finitely generated $R_{w}$-module for some $w(A) \subseteq w \subset W_{q}$. The $R_{w}$-action on $A$ induces an $R_{w}$-module structure on the set of $U$-valued points for any $\mathbb{F}_{q}$-scheme $U$. The fppf-sheafification $\left(M \otimes_{R_{w}} A\right)^{\#}$ of the functor on $\mathbb{F}_{q}$-schemes

$$
U \mapsto M \otimes_{R_{w}} A(U)
$$

is representable by an abelian variety. Indeed, let

$$
R_{w}^{m} \stackrel{\varphi}{\longrightarrow} R_{w}^{n} \longrightarrow M \longrightarrow 0
$$

be a finite presentation. The $m \times n$-matrix $\varphi$ also defines a map $\varphi_{A}: A^{m} \rightarrow A^{n}$, and $M \otimes_{R_{w}} A(U)=\operatorname{coker}\left(\varphi \otimes \operatorname{id}_{A(U)}\right)=\operatorname{coker}\left(\varphi_{A}: A(U)^{m} \rightarrow A(U)^{n}\right)=\operatorname{coker}\left(\varphi_{A}(U)\right)$, so that

$$
\left(M \otimes_{R_{w}} A\right)^{\#}=\operatorname{coker}\left(\varphi_{A}\right),
$$

and this is representable by an abelian variety. We denote the representing object by

$$
M \otimes_{R_{w}} A .
$$

If $w \subseteq w^{\prime}$ and $M^{\prime}$ is a finitely presented $R_{w^{\prime}}$-module with $M=M^{\prime} \otimes_{R_{w^{\prime}}} R_{w}$, then there is an obvious identification

$$
M^{\prime} \otimes_{R_{w^{\prime}}} A=M \otimes_{R_{w}} A .
$$

In particular, if $W \subseteq W_{q}$ is a subset and $w(A) \subseteq W$, then for any invertible $\mathscr{R}_{W^{-}}$ module $M=\left(M_{w}\right)$ we have a well-defined tensor product given by

$$
\mathcal{M} \otimes_{\mathscr{R}_{W}} A:=M_{w} \otimes_{R_{w}} A
$$

for all sufficiently large finite $w(A) \subseteq w \subseteq W$.

7.3. Choices of ind-representing objects. Before we describe our choices, we need three propositions of independent interest.

Proposition 34. Let $W \subseteq W_{q}$ be a subset, $A$ an abelian variety with $w(A) \subseteq W$, and $M=\left(M_{w}\right)$ an invertible $\mathscr{R}_{W}$-module. Then there is a natural isomorphism

$$
\operatorname{Hom}_{\mathbb{F}_{q}}\left(-, \mu \otimes_{\mathscr{R}_{W}} A\right) \simeq \mathcal{M} \otimes_{\mathscr{R}_{W}} \operatorname{Hom}_{\mathbb{F}_{q}}(-, A)
$$

of functors $\mathrm{AV}_{W} \rightarrow \operatorname{Refl}\left(\mathscr{R}_{W}\right)$. 
Proof. We set $w=w(A)$, and must show that naturally in $X$

$$
\operatorname{Hom}_{\mathbb{F}_{q}}\left(X, M_{w} \otimes_{R_{w}} A\right) \simeq M_{w} \otimes_{R_{w}} \operatorname{Hom}_{\mathbb{F}_{q}}(X, A)
$$

for any abelian variety $X$ over $\mathbb{F}_{q}$. We extend this claim to projective $R_{w}$-modules $M$ of finite rank. Since the tensor construction is compatible with direct sums, clearly the claim is additive in $M$ in the sense that it holds for $M^{\prime}$ and $M^{\prime \prime}$ if and only if it holds for $M=M^{\prime} \oplus M^{\prime \prime}$. This reduces the claim to free modules $M=R_{w}^{n}$, and by the same argument to $M=R_{w}$. Now the claim trivially holds.

Proposition 35. Let $W \subseteq W_{q}$ be a subset containing no rational Weil q-number. Any $\mathscr{R}_{W}$-linear contravariant equivalence

$$
S: \mathrm{AV}_{W} \rightarrow \operatorname{Refl}\left(\mathscr{R}_{W}\right)
$$

is ind-representable, i.e., of the form

$$
S(-)=\operatorname{Hom}_{\mathbb{F}_{p}}(-, \mathscr{B})
$$

for an ind-system $\mathscr{B}=\left(B_{w}, \varphi_{w, v}\right)$ such that the following holds for all finite subsets $v \subseteq w \subseteq W$ :

(i) $w\left(B_{w}\right)=w$.

(ii) The natural map $R_{w} \rightarrow \operatorname{End}_{\mathbb{F}_{q}}\left(B_{w}\right)$ is an isomorphism.

(iii) $B_{w}$ is isogenous to the product of its simple factors with multiplicity 1.

(iv) The maps $\varphi_{w, v}: B_{v} \rightarrow B_{w}$ are inclusions.

Proof. The pro-system $\mathscr{R}_{W}=\left(R_{w}, \mathrm{pr}_{v, w}\right)$ can be considered as the pro-system of the free rank-1 modules $R_{w} \in \operatorname{Refl}\left(R_{w}\right) \subseteq \operatorname{Refl}\left(\mathscr{R}_{W}\right)$. As such there is a unique ind-system $\mathscr{\mathscr { B }}=\left(B_{w}, \varphi_{w, v}\right)$ with $S(\mathscr{B})=\left(S\left(B_{w}\right)\right)=\mathscr{R}_{W}$. Yoneda's lemma assigns to the compatible elements $1 \in R_{w}=S\left(B_{w}\right)$ a natural transformation

$$
\Phi: \operatorname{Hom}_{\mathbb{F}_{q}}(-, \Re)=\underline{\lim _{w}} \operatorname{Hom}_{\mathbb{F}_{q}}\left(-, B_{w}\right) \rightarrow S(-) .
$$

For every $A \in \mathrm{AV}_{W}$ the map $\Phi$ is the composition of the two isomorphisms

$$
\underset{w}{\lim } \operatorname{Hom}\left(A, B_{w}\right) \stackrel{S}{\longrightarrow} \underset{w}{\lim } \operatorname{Hom}_{R_{w}}\left(R_{w}, S(A)\right) \stackrel{\mathrm{ev}_{1}}{\longrightarrow} S(A),
$$

where $\mathrm{ev}_{1}$ denotes the evaluation map at 1 . It remains to prove the finer claims on the ind-representing system $\mathscr{B}$.

Since $S$ is an $\mathscr{R}_{W}$-linear equivalence, $\mathscr{R}_{W}$ acts on $B_{w}$ through $R_{w}$ as on $S\left(B_{w}\right)=$ $R_{w}$. Here we use that $R_{w}$ is commutative, and so we can forget to pass to the opposite ring due to $S$ being contravariant. Since $F_{w}$ acts on $B_{w}$ by the Frobenius isogeny $\pi_{B_{w}}$, and on $R_{w}=S\left(B_{w}\right)$ by $F_{w} \in R_{w}$, it follows that $w\left(B_{w}\right)=w$. 
The natural map $R_{w} \rightarrow \operatorname{End}_{\mathbb{F}_{w}}\left(B_{w}\right)$ is an isomorphism, because applying the $\mathscr{R}_{W}$-linear $S(-)$ transforms it to the map $R_{w} \rightarrow \operatorname{End}_{\mathscr{R}_{w}}\left(R_{w}\right)$, which is indeed an isomorphism. We deduce assertion (iii) from this as well.

It remains to show that $\varphi_{w, v}: B_{v} \rightarrow B_{w}$ is isomorphic to an inclusion for all $v \subseteq w$. We denote the image of $\varphi_{w, v}$ by $C$. Since $S$ is ind-representable, the surjection $B_{v} \rightarrow C$ becomes an inclusion

$$
S(C) \hookrightarrow S\left(B_{v}\right) .
$$

Since by construction $S\left(B_{w}\right) \rightarrow S\left(B_{v}\right)$ is the surjective map $\operatorname{pr}_{v, w}: R_{w} \rightarrow R_{v}$, we conclude that $S(C) \simeq S\left(B_{v}\right)$ is an isomorphism. Consequently, because $S$ is an equivalence, we have $C \simeq B_{v}$ and assertion (iv) holds.

The third proposition is related to Proposition 24.

Proposition 36. Let $W \subseteq W_{q}$ be a subset containing no rational Weil q-number, and let

$$
S: \mathrm{AV}_{W} \rightarrow \operatorname{Refl}\left(\mathscr{R}_{W}\right)
$$

be an $\mathscr{R}_{W}$-linear contravariant equivalence.

Let $w \subseteq W$ be a finite set of conjugacy classes of Weil $q$-numbers, and let $A$ be an abelian variety over $\mathbb{F}_{q}$ with $w=w(A)$. The following are equivalent:

(a) The natural map $R_{w} \rightarrow \operatorname{End}_{\mathbb{F}_{q}}(A)$ is an isomorphism.

(b) $S(A)$ is a projective $R_{w}$-module of rank 1 .

Proof. Since $S(-)$ is an equivalence of categories, the map $R_{w} \rightarrow \operatorname{End}_{\mathbb{F}_{p}}(A)$ is an isomorphism if and only if the map

$$
R_{w} \rightarrow \operatorname{End}_{R_{w}}(S(A))
$$

is an isomorphism ( $S$ is contravariant but the rings are commutative here). Since $R_{w}$ is a reduced Gorenstein ring of dimension 1 by Theorem 11, this is equivalent by Proposition 18 to $S(A)$ being a projective $R_{w}$-module of rank 1 .

We define the tensor product of an invertible $\mathscr{R}_{W}$-module $\mathcal{M}=\left(M_{w}\right)$ and an ind-system $\mathscr{A}=\left(A_{w}, \varphi_{w, v}\right)$ of abelian varieties indexed by finite subsets of $W$ and with $w\left(A_{w}\right)=w$ by

$$
M \otimes \mathscr{A}:=\left(M_{w} \otimes_{R_{w}} A_{w}\right) .
$$

Theorem 37. Let $W \subseteq W_{q}$ be a subset containing no rational Weil q-number.

Let $\mathscr{A}=\left(A_{w}, \varphi_{w, v}\right)$ be an ind-system of abelian varieties over $\mathbb{F}_{q}$ indexed by finite subsets of $W$ such that:

(i) $w\left(A_{w}\right)=w$.

(ii) The natural map $R_{w} \rightarrow \operatorname{End}_{\mathbb{F}_{q}}\left(A_{w}\right)$ is an isomorphism. 
(iii) $A_{w}$ is isogenous to the product of its simple factors with multiplicity 1.

(iv) The maps $\varphi_{w, v}: A_{v} \rightarrow A_{w}$ are inclusions.

For an invertible $\mathscr{R}_{W}$-module $M$, the ind-system $M \otimes_{\mathscr{R}_{W}} \mathscr{A}$ has the same properties (i)-(iv), and the group $\operatorname{Pic}\left(\mathscr{R}_{W}\right)$ acts freely and transitively by

$$
\mathscr{A} \mapsto M \otimes_{\mathscr{R}_{W}} \mathscr{A}
$$

on the set of isomorphism classes of such ind-systems.

Remark 38. When $q=p$ and $W=\{\pi\}$ consists of a single Weil $p$-number, Theorem 37 is a special case of [Waterhouse 1969, Theorem 6.1.3], which inspired the above result.

Proof of Theorem 37. By a $W$-version of the proof of Theorem 1 for any ind-system $\mathscr{A}$ satisfying (i)-(iv), the functor

$$
T_{\mathscr{A}}=\operatorname{Hom}_{\mathbb{F}_{q}}(-, \mathscr{A}): \mathrm{AV}_{W} \rightarrow \operatorname{Refl}\left(\Re_{W}\right)
$$

is a contravariant $\mathscr{R}_{W}$-linear antiequivalence $\mathrm{AV}_{W} \rightarrow \operatorname{Refl}\left(\mathscr{R}_{W}\right)$. The effect of the action by $M \in \operatorname{Pic}\left(\mathscr{R}_{W}\right)$ on the represented functors is described by Proposition 34 as

$$
T_{M \otimes \mathscr{R}_{W}} \mathscr{A}(-)=\mathcal{M} \otimes_{\mathscr{R}_{W}} T_{\mathscr{A}}(-) .
$$

Since $\mathcal{M}=\left(M_{w}\right)$ is invertible, the functor $\mathcal{M} \otimes_{\mathscr{R}_{W}}-$ is an autoequivalence of $\mathrm{AV}_{W}$. We thus have natural isomorphisms

$$
R_{w}=\operatorname{End}_{\mathbb{F}_{q}}\left(A_{w}\right)=\operatorname{End}_{\mathbb{F}_{q}}\left(\mathcal{M} \otimes_{\mathscr{R}_{W}} A_{w}\right)=T_{\mathcal{M} \otimes_{\mathscr{R}_{W}} \mathscr{A}}\left(\mathcal{M} \otimes_{\mathscr{R}_{W}} A_{w}\right) .
$$

Moreover, since $M=\left(M_{w}\right)$ is invertible, the functor $T_{M \otimes_{\mathscr{R}_{W}}}(-)$ is an antiequivalence as well, and

$$
T_{\mathcal{M} \otimes_{\mathscr{R}_{W}} \mathscr{A}}\left(\mathcal{M} \otimes_{\mathscr{R}_{W}} \mathscr{A}\right)=\mathscr{R}_{W}
$$

as pro-systems. It follows from the proof of Proposition 35 that $M \otimes_{\mathscr{R}_{W}} \mathscr{A}$ also satisfies properties (i)-(iv). This shows that $\operatorname{Pic}\left(\mathscr{R}_{W}\right)$ indeed acts on isomorphism classes of such $\mathscr{A}$.

Let $\mathcal{M}$ be an invertible $\mathscr{R}_{W}$-module, and let $\mathscr{A}$ be a pro-system as above such that there is an isomorphism $M \otimes_{\mathscr{R}_{W}} \mathscr{A} \simeq \mathscr{A}$. Evaluating the resulting natural isomorphism

$$
\mathcal{M} \otimes_{\mathscr{R}_{W}} T_{\mathscr{A}}(-) \simeq T_{\mathscr{A}}(-)
$$

in $\mathscr{A}$ itself yields an isomorphism $M \otimes_{\mathscr{R}_{W}} \mathscr{R}_{W} \simeq \mathscr{R}_{W}$, and hence $\mathcal{M}$ must be trivial in $\operatorname{Pic}\left(\mathscr{R}_{W}\right)$. This shows that the action is free.

Let now $\mathscr{A}$ and $\mathscr{B}$ be two pro-systems of the type considered. The $\mathscr{R}_{W}$-module

$$
\mathcal{M}=T_{\mathscr{B}}(\mathscr{A})=\left(\operatorname{Hom}_{\mathbb{F}_{p}}\left(A_{w}, B_{w}\right)\right)
$$


(note that all maps of pro-objects $\mathscr{A} \rightarrow \mathscr{B}$ are levelwise maps since $w\left(A_{w}\right)=w=$ $\left.w\left(B_{w}\right)\right)$ is levelwise an invertible $R_{w}$-module $\mu_{w}=T_{\mathscr{R}}\left(A_{w}\right)$ by Proposition 36. The transfer maps $\mathcal{M}_{w} \rightarrow \mathcal{M}_{v}$ agree with $T_{\mathscr{B}}\left(\varphi_{w, v}\right)$, which is surjective. Indeed, the image corresponds to an abelian variety $C$ such that $\varphi_{w, v}$ factors as

$$
A_{v} \longrightarrow C \longrightarrow A_{w} \text {. }
$$

Now the same argument as in the proof of Proposition 35 shows that $w(C) \subseteq w$ and $C \rightarrow A_{w}$ is an inclusion. Since $\varphi_{w, v}$ is an inclusion, we necessarily have $A_{v}=C$ and $T_{\mathscr{R}}\left(\varphi_{w, v}\right)$ is indeed surjective. Consequently, the $\mathscr{R}_{W}$-module $\mathcal{M}=\left(\mathcal{M}_{w}\right)$ is invertible.

There is a natural map defined by composition of maps

$$
M \otimes_{\mathscr{R}_{W}} T_{\mathscr{A}}(-)=\operatorname{Hom}(\mathscr{A}, \mathscr{B}) \otimes \operatorname{Hom}(-, \mathscr{A}) \rightarrow \operatorname{Hom}(-, \mathscr{B})=T_{\mathscr{B}}(-) .
$$

This is an isomorphism, because for every $X$ in $\mathrm{AV}_{W}$ and large enough $w$ we have

$$
\begin{aligned}
\mu \otimes_{\mathscr{R}_{W}} T_{\mathscr{A}}(X) & =\operatorname{Hom}_{\mathbb{F}_{p}}\left(A_{w}, B_{w}\right) \otimes_{R_{w}} \operatorname{Hom}_{\mathbb{F}_{p}}\left(X, A_{w}\right) \\
& =T_{\mathscr{B}}\left(A_{w}\right) \otimes_{R_{w}} \operatorname{Hom}_{R_{w}}\left(T_{\mathscr{B}}\left(A_{w}\right), T_{\mathscr{B}_{B}}(X)\right) \\
& =T_{\mathscr{R}}(X) .
\end{aligned}
$$

Here we have used again the assumption that $T_{\mathscr{B}}(-)$ is an equivalence and the fact that $T_{\mathscr{B}}\left(A_{w}\right)$ is invertible as an $R_{w}$-module by Proposition 36 .

Corollary 39. The action of $\mathrm{Pic}\left(\mathscr{R}_{p}^{\mathrm{com}}\right)$ on the isomorphism classes of ind-systems $A$ that represent $\mathscr{R}_{p}^{\mathrm{com}}$-linear antiequivalences $\mathscr{A}_{p}^{\mathrm{com}} \rightarrow \operatorname{Refl}\left(\mathscr{R}_{p}^{\mathrm{com}}\right)$ is free and transitive.

Proof. This follows immediately from Theorem 37, the proof of Theorem 1 and Proposition 35.

Remark 40. With the notation of Section 5.3, for finite sets $v \subseteq w \subseteq W_{p}$ avoiding $\sqrt{p}$ the transfer map

$$
\zeta_{v, w}: Z(w) \rightarrow Z(v)
$$

in the pro-system of isomorphism classes occurring in the proof of Theorem 1 is in fact surjective. This follows immediately from Theorem 37 and the surjectivity of $\operatorname{Pic}\left(R_{w}\right) \rightarrow \operatorname{Pic}\left(R_{v}\right)$ from Proposition 33 .

Corollary 41. Let $V \subseteq W \subseteq W_{p}$ be subsets avoiding $\sqrt{p}$, and let $\mathscr{A}_{V}=\left(A_{v}, \varphi_{w, v}\right)$ be an ind-system of abelian varieties over $\mathbb{F}_{p}$ indexed by finite subsets of $V$ as in Theorem 37 such that

$$
T_{\mathscr{A}_{V}}=\operatorname{Hom}_{\mathbb{F}_{p}}\left(-, \mathscr{A}_{V}\right): \mathrm{AV}_{V} \rightarrow \operatorname{Refl}\left(\mathscr{R}_{V}\right)
$$


is an $\mathscr{R}_{V}$-linear antiequivalence of categories. Then $\mathscr{A}_{V}$ can be extended to an ind-system $\mathscr{A}_{W}=\left(A_{w}, \varphi_{v, w}\right)$ of abelian varieties over $\mathbb{F}_{p}$ indexed by finite subsets of $W$ as in Theorem 37. In particular the antiequivalence

$$
T_{\mathscr{A}_{W}}=\operatorname{Hom}_{\mathbb{F}_{p}}\left(-, \mathscr{A}_{W}\right): \mathrm{AV}_{W} \rightarrow \operatorname{Refl}\left(\mathscr{R}_{W}\right)
$$

naturally extends $T_{\mathscr{A}_{V}}$.

Proof. We start by choosing an auxiliary ind-system $\mathscr{B}_{W}$ indexed by finite subsets of $W$ as in Theorem 37. The restriction

$$
\operatorname{Hom}_{\mathbb{F}_{p}}\left(-, \mathscr{B}_{W}\right): \mathrm{AV}_{V} \rightarrow \operatorname{Refl}\left(\mathscr{R}_{V}\right)
$$

is an $\mathscr{R}_{V}$-linear antiequivalence and is ind-represented by the restriction $\mathscr{B}_{V}=\left.\mathscr{B}_{W}\right|_{V}$ of the indices to finite subsets of $V$. By Theorem 37 there is an $\mathcal{M}_{V} \in \operatorname{Pic}\left(\mathscr{R}_{V}\right)$ such that

$$
\mathscr{A}_{V}=\mathcal{M}_{V} \otimes_{\mathscr{R}_{V}} \mathscr{B}_{V}
$$

By Proposition 33 we can find $\mathcal{M}_{W} \in \operatorname{Pic}\left(\mathscr{R}_{W}\right)$ such that $\mathcal{M}_{V}=\mathcal{M}_{W} \otimes_{\mathscr{R}_{W}} \mathscr{R}_{V}$. Then

$$
\mathscr{A}_{W}=\mathcal{M}_{W} \otimes_{\mathscr{R}_{w}} \Re_{W}
$$

obviously extends $\mathscr{A}_{V}$ in the desired manner.

7.4. Comparison with Deligne's functor for ordinary abelian varieties over $\mathbb{F}_{p}$. Let $w \subseteq W_{p}^{\text {com }}$ be a finite subset, and let $\tau: R_{w} \rightarrow R_{w}$ be the automorphism interchanging $F_{w}$ and $V_{w}$. Denote by $R_{w}^{\tau}$ the $R_{w}$-module obtained by letting $R_{w}$ operate onto itself via $\tau$. Similarly, for an object $M$ of $\operatorname{Refl}\left(R_{w}\right)$, denote by $M^{\tau}$ the $R_{w}$-module $M \otimes_{R_{w}} R_{w}^{\tau}$.

We fix a contravariant equivalence $T$ as in Theorem 1, and an ind-representing system $\mathscr{A}=\left(A_{w}, \varphi_{w^{\prime}, w}\right)$ for $T=T_{\mathscr{A}}$. The covariant functor on $\mathrm{A \bigvee}_{p}^{\text {com }}$

$$
T_{*}(A)=T\left(A^{t}\right)^{\tau}=\underset{w}{\lim } \operatorname{Hom}\left(A_{w}^{t}, A\right),
$$

is pro-representable by the dual system $\mathscr{A}^{t}=\left(A_{w}^{t}, \varphi_{w^{\prime}, w}^{t}\right)$ and a version of Theorem 1 with a covariant equivalence

$$
T_{*}: \mathrm{AV}_{p}^{\mathrm{com}} \rightarrow \operatorname{Refl}\left(\mathscr{R}_{p}^{\mathrm{com}}\right)
$$

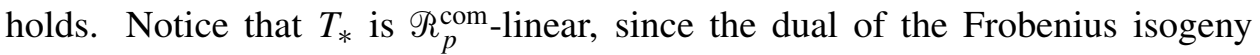
$\pi_{A}: A \rightarrow A$ is the Verschiebung isogeny $p / \pi_{A^{t}}: A^{t} \rightarrow A^{t}$.

We recall that Deligne's functor $T_{\text {Del }}$ on $\mathrm{AV}_{q}^{\text {ord }}$ is defined as

$$
T_{\text {Del }}(A)=\mathrm{H}_{1}(\tilde{A}(\mathbb{C}), \mathbb{Z}),
$$

where $\widetilde{A} / W\left(\overline{\mathbb{F}}_{p}\right)$ is the Serre-Tate canonical lift of $A \otimes_{\mathbb{F}_{q}} \overline{\mathbb{F}}_{p}$ to characteristic 0 over the Witt-vectors $W\left(\overline{\mathbb{F}}_{p}\right)$, and where the $\mathbb{C}$-valued points are taken with respect to an 
a priori fixed embedding $W\left(\overline{\mathbb{F}}_{p}\right) \hookrightarrow \mathbb{C}$. The lattice $T_{\text {Del }}(A)$ comes equipped with a natural Frobenius action by $F=T_{\mathrm{Del}}\left(\pi_{A}\right)$.

Note that the functor depends on the chosen embedding $W\left(\overline{\mathbb{F}}_{p}\right) \hookrightarrow \mathbb{C}$.

We denote by $W_{q}^{\text {ord }}$ the set of conjugacy classes of ordinary Weil $q$-numbers, i.e., of Weil $q$-numbers such that at least half of the roots of the characteristic polynomial are $p$-adic units, when regarded inside an algebraic closure of $\mathbb{Q}_{p}$. With the abbreviation $\mathscr{R}_{q}^{\text {ord }}=\mathscr{R}_{W_{q}^{\text {ord }}}$, the main result of [Deligne $1969, \S 7$ ] can be stated as:

Theorem 42. The covariant functor $T_{\text {Del }}$ induces an $\mathscr{R}_{q}^{\text {ord }}$-linear equivalence of categories

$$
T_{\text {Del }}: \mathrm{AV}_{q}^{\text {ord }} \rightarrow \operatorname{Refl}\left(\mathscr{R}_{q}^{\text {ord }}\right) .
$$

We now compare $T_{*}(-)$ with $T_{\mathrm{Del}}$ when both are restricted to $\mathrm{AV}_{p}^{\text {ord: }}$

Proposition 43. The functor $T_{\mathrm{Del}}(-)$ is pro-representable by a pro-system $\mathscr{A}_{\mathrm{Del}}$ and

$$
T_{\text {Del }}\left(\mathscr{A}_{\text {Del }}\right)=\mathscr{R}_{q}^{\text {ord }}
$$

The dual ind-system $\mathscr{A}_{\text {Del }}^{t}$ satisfies (i)-(iv) of Proposition 35.

Proof. This follows from Proposition 35 applied to the functor $X \mapsto T_{\text {Del }}\left(X^{t}\right)$.

Let $T_{*}^{\text {ord }}$ and $T_{\mathrm{Del}, p}$ denote the restriction of $T_{*}$ and $T_{\mathrm{Del}}$ to $\mathrm{AV}_{p}^{\text {ord }}$, respectively. The functor $T_{*}^{\text {ord }}$ is pro-represented by the dual $\mathscr{A}^{\text {ord, } t}$ of the ind-system $\mathscr{A}^{\text {ord }}$ which is defined as $\mathscr{A}$ restricted to indices in $W_{p}^{\text {ord }}$.

Proposition 44. There is an invertible $\mathscr{R}_{p}^{\text {ord }}$-module $\mathcal{M}=\left(M_{w}\right)_{w \in W_{p}^{\text {ord }}}$ and a natural isomorphism

$$
M \otimes_{\mathscr{R}_{p}^{\text {ord }}} T_{\mathrm{Del}, p}(-) \stackrel{\sim}{\longrightarrow} T_{*}^{\text {ord }}(-)
$$

of covariant equivalences $\mathrm{AV}_{p}^{\text {ord }} \rightarrow \operatorname{Refl}\left(\Re_{p}^{\text {ord }}\right)$, and a natural isomorphism of ind-systems

$$
M \otimes_{\mathscr{R}_{q}^{\text {ord }}} \mathscr{A}_{\text {Del }}^{t} \simeq \mathscr{A}^{\text {ord }} .
$$

Proof. This follows from Theorem 37 applied to $W=W_{q}^{\text {ord }}$.

Proposition 45. For an appropriate choice of ind-system $\mathscr{A}=\left(A_{w}, \varphi_{v, w}\right)$, the covariant functor $T_{*}$ associated to the functor $T=T_{\mathscr{A}}$ of Theorem 1 extends a given choice of Deligne's functor

$$
\left.T_{\mathrm{Del}, p} \simeq T_{*}\right|_{\mathscr{A}_{p}^{\text {ord }}}: \mathrm{AV}_{p}^{\text {ord }} \rightarrow \operatorname{Refl}\left(\mathscr{R}_{p}^{\text {ord }}\right) .
$$

Proof. This follows from Proposition 44 together with the argument of Corollary 41 based on the surjectivity $\operatorname{Pic}\left(\mathscr{R}_{p}^{\text {com }}\right) \rightarrow \operatorname{Pic}\left(\Re_{p}^{\text {ord }}\right)$ of Proposition 33. 


\section{Acknowledgments}

The authors would like to thank Gebhard Böckle for stimulating discussions and for his suggestion of the symmetric polynomial $h_{\pi}(F, V)$. We thank Filippo Nuccio for valuable comments on an earlier version of the manuscript, and Hendrik Lenstra for his interesting observations on local complete intersections. Special thanks go to Brian Conrad and Frans Oort for their attentive reading of a preliminary version of our work, and for the prompt and interesting feedback they gave us. Finally we thank the anonymous referees for their quick work in reviewing the paper.

\section{References}

[Bass 1963] H. Bass, "On the ubiquity of Gorenstein rings", Math. Z. 82 (1963), 8-28. MR 27 \#3669 Zbl 0112.26604

[Chai et al. 2014] C.-L. Chai, B. Conrad, and F. Oort, Complex multiplication and lifting problems, Mathematical Surveys and Monographs 195, Amer. Math. Soc., Providence, RI, 2014. MR 3137398 Zbl 1298.14001

[Deligne 1969] P. Deligne, "Variétés abéliennes ordinaires sur un corps fini”, Invent. Math. 8 (1969), 238-243. MR 40 \#7270 Zbl 0179.26201

[Giraud 1968] J. Giraud, "Remarque sur une formule de Shimura-Taniyama”, Invent. Math. 5 (1968), 231-236. MR 37 \#2757 Zbl 0165.54801

[Howe 1995] E. W. Howe, "Principally polarized ordinary abelian varieties over finite fields", Trans. Amer. Math. Soc. 347:7 (1995), 2361-2401. MR 96i:11065 Zbl 0859.14016

[Howe 2004] E. W. Howe, "On the non-existence of certain curves of genus two", Compos. Math. 140:3 (2004), 581-592. MR 2005a:11088 Zbl 1067.11035

[Matsumura 1989] H. Matsumura, Commutative ring theory, 2nd ed., Cambridge Studies in Advanced Mathematics 8, Cambridge University Press, 1989. MR 90i:13001 Zbl 0666.13002

[Milne 1986] J. S. Milne, "Abelian varieties", pp. 103-150 in Arithmetic geometry (Storrs, CT, 1984), edited by G. Cornell and J. H. Silverman, Springer, New York, 1986. MR 861974 Zbl 0604.14028

[Serre and Tate 1968] J.-P. Serre and J. Tate, "Good reduction of abelian varieties", Ann. of Math. (2) 88 (1968), 492-517. MR 38 \#4488 Zbl 0172.46101

[Tate 1966] J. Tate, "Endomorphisms of abelian varieties over finite fields", Invent. Math. 2 (1966), 134-144. MR 34 \#5829 Zbl 0147.20303

[Tate 1971] J. Tate, "Classes d'isogénie des variétés abéliennes sur un corps fini (d'après T. Honda)", exposé no. 352, 95-110 in Séminaire Bourbaki, 1968/69, Lecture Notes in Math. 175, Springer, Berlin, 1971. MR 3077121 Zbl 0212.25702

[Waterhouse 1969] W. C. Waterhouse, "Abelian varieties over finite fields", Ann. Sci. École Norm. Sup. (4) 2 (1969), 521-560. MR 42 \#279 Zbl 0188.53001

[Waterhouse and Milne 1971] W. C. Waterhouse and J. S. Milne, "Abelian varieties over finite fields", pp. 53-64 in 1969 Number Theory Institute (Proc. Sympos. Pure Math., Vol. XX, State Univ. New York, Stony Brook, NY., 1969), edited by D. Lewis, Amer. Math. Soc., Providence, R.I., 1971. MR 47 \#3397 Zbl 0216.33102

Communicated by Brian Conrad

Received 2014-07-21 Revised 2014-12-05 Accepted 2015-01-06 
tommaso.centeleghe@iwr.uni-heidelberg.de

IWR, Universität Heidelberg, Im Neuenheimer Feld 368, D-69120 Heidelberg, Germany

stix@math.uni-heidelberg.de Institut für Mathematik, Goethe-Universität Frankfurt, Robert-Mayer-Straße 6-8, D-60325 Frankfurt am Main, Germany 


\section{Algebra \& Number Theory}

msp.org/ant

\section{EDITORS}

MANAGING EDITOR

Bjorn Poonen

Massachusetts Institute of Technology

Cambridge, USA

\author{
EDITORIAL BOARD CHAIR \\ David Eisenbud \\ University of California \\ Berkeley, USA
}

BOARD OF EDITORS

Georgia Benkart

Dave Benson

Richard E. Borcherds

John H. Coates

J-L. Colliot-Thélène

Brian D. Conrad

Hélène Esnault

Hubert Flenner

Edward Frenkel

Andrew Granville

Joseph Gubeladze

Roger Heath-Brown

Craig Huneke

János Kollár

Yuri Manin

Barry Mazur

Philippe Michel

Susan Montgomery
University of Wisconsin, Madison, USA

University of Aberdeen, Scotland

University of California, Berkeley, USA

University of Cambridge, UK

CNRS, Université Paris-Sud, France

University of Michigan, USA

Freie Universität Berlin, Germany

Ruhr-Universität, Germany

University of California, Berkeley, USA

Université de Montréal, Canada

San Francisco State University, USA

Oxford University, UK

University of Virginia, USA

Princeton University, USA

Northwestern University, USA

Harvard University, USA

École Polytechnique Fédérale de Lausanne

University of Southern California, USA
Shigefumi Mori

Raman Parimala

Jonathan Pila

Anand Pillay

Victor Reiner

Peter Sarnak

Joseph H. Silverman

Michael Singer

Vasudevan Srinivas

J. Toby Stafford

Richard Taylor

Ravi Vakil

Michel van den Bergh

Marie-France Vignéras

Kei-Ichi Watanabe

Efim Zelmanov

Shou-Wu Zhang
RIMS, Kyoto University, Japan

Emory University, USA

University of Oxford, UK

University of Notre Dame, USA

University of Minnesota, USA

Princeton University, USA

Brown University, USA

North Carolina State University, USA

Tata Inst. of Fund. Research, India

University of Michigan, USA

Harvard University, USA

Stanford University, USA

Hasselt University, Belgium

Université Paris VII, France

Nihon University, Japan

University of California, San Diego, USA

Princeton University, USA

\section{PRODUCTION}

production@msp.org

Silvio Levy, Scientific Editor

See inside back cover or msp.org/ant for submission instructions.

The subscription price for 2015 is US \$255/year for the electronic version, and $\$ 440 /$ year ( $\$ 55$, if shipping outside the US) for print and electronic. Subscriptions, requests for back issues and changes of subscribers address should be sent to MSP.

Algebra \& Number Theory (ISSN 1944-7833 electronic, 1937-0652 printed) at Mathematical Sciences Publishers, 798 Evans Hall \#3840, c/o University of California, Berkeley, CA 94720-3840 is published continuously online. Periodical rate postage paid at Berkeley, CA 94704, and additional mailing offices.

ANT peer review and production are managed by EditFLOW ${ }^{\circledR}$ from MSP.

\section{PUBLISHED BY}

7 mathematical sciences publishers

nonprofit scientific publishing

http://msp.org/

(C) 2015 Mathematical Sciences Publishers 


\section{Algebra \& Number Theory}

Volume $9 \quad$ No. $1 \quad 2015$

On Previdi's delooping conjecture for $K$-theory

SHo SAITO

Surpassing the ratios conjecture in the 1-level density of Dirichlet $L$-functions

DANIEL FIORILli and STEVEN J. MiLler

Eisenstein Hecke algebras and conjectures in Iwasawa theory

PRESTON WAKE

Adequate groups of low degree

Robert Guralnick, Florian Herzig and Pham HuU TieP

Random matrices, the Cohen-Lenstra heuristics, and roots of unity

DEREK GARTON

Local Beilinson-Tate operators

173

AMNON YEKUTIELI

Categories of abelian varieties over finite fields, I: Abelian varieties over $\mathbb{F}_{p}$ 